\title{
Aerothermoelastic Reduced-Order Model of a Hypersonic Vehicle
}

\author{
Ryan J. Klock ${ }^{1}$ and Carlos E.S. Cesnik ${ }^{2}$ \\ University of Michigan, Ann Arbor, MI 48109-2140
}

Model reduction techniques are applied to a hypersonic vehicle on terminal trajectories to capture the aerodynamic, thermodynamic, and structural dynamic system evolution and couplings. The General Pseudospectral Optimization Software (GPOPS-II) was used to determine a set of terminal trajectories which maximized impact velocity. Shock, Prandtl-Meyer expansion, and piston theory were combined to create an approximate flow solution over the outer mold line which was then compared to Fully Unstructured Navier-Stokes 3-Dimensional (FUN3D) computational fluid dynamics solutions. Proper orthogonal decomposition of the thermal state of the vehicle was conducted leading to 33 thermal degrees of freedom rather than approximately 28,000 contained by a representative finite element model, while sacrificing negligible system energy. Free vibration mode shapes are derived and used to generalize the structural dynamics equations of motion reducing the number of structural degrees of freedom to 8 from the original 130,000 . Finally, the combination of these reduced order models is discussed in the context of future work toward a full vehicle simulation.

\section{Introduction}

$\mathrm{H}$ IGH speed weapons systems, particularly hypersonic vehicles, operate in a high energy environment characterized by strong fluid, thermal, and structural interactions. Due to a lack of ground test facilities which can generate the high energy environment of interest, the primary course of investigation must be through computational simulations. Current computational research efforts have focused on either improving particular physics model fidelity with limited discipline interactions due to a high computational cost, or including many discipline interactions using very simple models. Thus, there is a wide middle-ground between the low-fidelity, high-interaction and high-fidelity, low-interaction modeling regimes that has yet to be considered, but is critical to the development of high speed weapons systems. To bridge the modeling middle-ground, this study aimed to develop a set of reduced-order models (ROMs) to describe the thermal and structural interaction based on highfidelity finite element modeling analysis.

This included first establishing a representative vehicle configuration and trajectory. An initial study at the Eglin Air Force Base Munitions Directorate lead to the creation of a representative configuration by using the Preliminary Aerothermal Structural Simulation (PASS) code suite. ${ }^{1}$ This vehicle was to be an air-launched, rocket-scramjet combined cycle propelled vehicle which performed a three phase trajectory. Shown in Figure 1, the vehicle would first boost under rocket propulsion up to a cruising altitude of more than $50 \mathrm{kft}(15.2 \mathrm{~km})$ and Mach number greater than 5. The spent rocket booster would then be jettisoned and a scramjet propulsion system engaged to maintain a cruise condition for the majority of the overall trajectory. Finally, after exhausting the scramjet fuel supply, the vehicle would enter a terminal phase in a hypersonic glide condition to reach a ground target some distance downrange.

Witeof and Neergaard ${ }^{2}$ performed material trade and sizing optimization studies of thermal protection systems (TPS) and structural elements to minimize mass while satisfying material temperature and stiffness constraints. In their trade study, aerodynamic heating was approximated using the Aerothermal Target Analysis Program (ATAP) ${ }^{3}$ along a simulated boost-cruise-terminal mission profile representative of air-launched, rocket-boosted hypersonic vehicles and depicted in Figure 1. Structural modes were approximated using Timoshenko beam elements and the variation in modal frequencies due to thermal effects was investigated with respect to flight time.

\footnotetext{
${ }_{1}^{1}$ Graduate Research Assistant, Department of Aerospace Engineering, AIAA Student Member, rjklock@umich.edu

${ }^{2}$ Professor, Department of Aerospace Engineering, AIAA Fellow, cesnik@umich.edu
}

1

American Institute of Aeronautics and Astronautics

DISTRIBUTION A. Approved for public release, distribution unlimited. (96TW-2015-0158) 


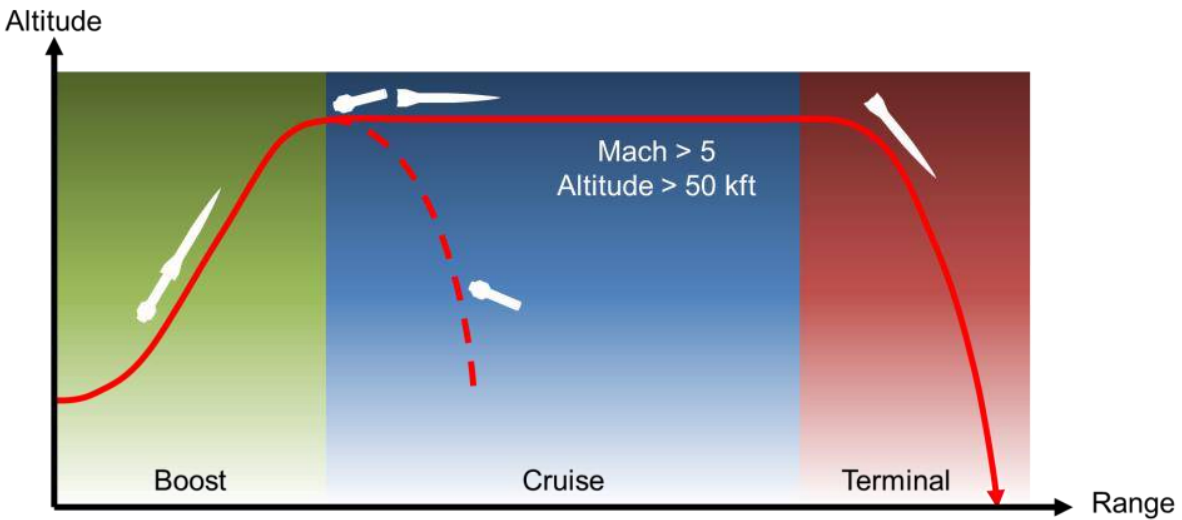

Figure 1: Basic outline of a boost-cruise-terminal mission profile for an air-launched, rocket-boosted hypersonic vehicle

For this study, we were interested in the terminal portion of the mission profile and required a representative terminal trajectory for which to tailor structural, thermal, and thermo-structural ROMs. In the literature, the optimization of hypersonic trajectories for a wide range of vehicle and mission types has been approached using many different optimization tools. Zhao and Zhou employed a multiple phase Gauss Pseudospectral method to maximize cross range and minimize trajectory time for a reentering hypersonic vehicle subjected to heating, loading, waypoint, and no-fly zone constraints. This was done by partitioning the trajectory into segments with matching final/initial conditions at the segment interfaces to create a continuous trajectory while refining the Legendre-Gauss sample density around periods of constraint influence. Rao and Clarke ${ }^{5}$ used the Legendre Pseudospectral method to similarly optimize a hypersonic reentry trajectory, however with a focus on maximizing control margin which would allow for unmodeled perturbations to the vehicle during an actual flight. The possibility of using the pseudospectral method as a guidance law was also discussed. An intelligent method based on multi-objective particle swarm optimization was developed by Grant and Mendeck ${ }^{6}$ to explore the design space of the Mars Science Laboratory entry trajectory in terms of parachute deployment altitude, range error, and acceleration loading. This method was especially well-suited to handle Pareto fronts, solution loci in the design space along which even trade-offs occur between multiple objective functions and provide no single superior solution. Also available is the Optimal Trajectories by Implicit Simulation (OTIS) software by Hargraves et al. ${ }^{7}$ which can generate open-loop control functions based on Hermite interpolation of the trajectory and nonlinear programming. Many types of point-mass and rigid-body problems have been considered using the OTIS tools including single- and multi-stage-to-orbit hypersonic vehicle trajectories. Other trajectory solvers are described in Refs. 8-10.

For the work presented here, the General Pseudospectral Optimization Software (GPOPS-II) was used to apply the Radau Pseudospectral method, ${ }^{11}$ previously used by Rexius et al. ${ }^{12}$ to optimize the launch, staging, and descent of a rocket-boosted hypersonic glider similar to the common aero vehicle (CAV). ${ }^{13}$ GPOPS-II was viewed by the authors as a suitable trajectory optimizer due to similar flight constraints during the final descent phase of the CAV presented by Rexius and the vehicle considered in this work, as well as the ease of use due to its Matlab based implementation.

It is often noted in literature that full-order simulation, particularly CFD and FEA for aerodynamic, structural dynamic, and thermodynamic coupled systems, can be prohibitively costly in terms of computational reasources. ${ }^{14}$, 15, 16, 17 To reduce the computational cost associated with obtaining a thermal solution for a vehicle structure using traditional finite element analysis, ROMs are often employed. A straightforward ROM approach is to use eigenmode analysis as described by Shore, ${ }^{18}$ which is analogous to the derivation of free-vibration modes for a structure, except that thermal conductivity and capacity are considered in place of the stiffness and inertia matrices. This leads to a set of basis modes from which a subset of temperature distributions may be selected and used as generalized degrees of freedom of the thermal problem. This approach may be extended with the component mode synthesis method described by Craig and Bampton. ${ }^{19}$ In component mode synthesis, a structure is first partitioned into a set of interfacing substructures. Each substructure's eigenmodes are determined with fully constrained boundary conditions at the interfaces to form a basis set for each substructure. The basis sets are then augmented with boundary modes derived from perturbation of the substructure interfaces which are selected to ensure continuity between substructures. By reducing the order of the basis sets of each substructure, the number of degrees of

2

American Institute of Aeronautics and Astronautics

DISTRIBUTION A. Approved for public release, distribution unlimited. (96TW-2015-0158) 
freedom of the overall structure may be reduced. This approach is useful for structures with a small number of components that have dissimilar properties, but can be cumbersome as the number of interfaces increases, requiring more and more boundary modes. The Guyan reduction method ${ }^{20}$ is possible if the thermal loading locations are known a priori and may be used to construct matrices whose entries only pertain to degrees of freedom that are known to vary and yet sacrifices none of the structural complexity. This approach is useful for thermal problems in which there are a few localized heat sources, but is poor at reducing the model complexity in scenarios with widely distributed loads, such as aerodynamic heating of a vehicle. Another possible technique to reduce the thermal problem is the modal identification method. ${ }^{21}$ In this approach, a set of eigenmodes of a state-space representation of the full-order system are identified through minimization of a criterion related to the difference between the outputs of the full and reduced-order systems. This is useful when a full eigenmode analysis of the state-space is computational infeasible and the thermal conductivity and capacity matrices are unknown. However, for this work, the thermal matrices will be known and thus, the advantage is inconsequential. Furthermore, the modal identification method has been shown to be effective for systems with low numbers of thermal loads (1 to 3 ), but will become infeasible for the thousands of loads considered over the entire outer surface of a hypersonic vehicle. ${ }^{22}$

Nearly all of these ROMs involve the transformation of a governing system of equations into modal space and differ in the identification of the basis set and correction for nonlinear effects. However, these methods largely rely on the matrices of the governing systems of equations rather than the actual response of the system when observed during simulation or experimentation. Furthermore, eigenvector bases may not be optimal in the sense of capturing the most system energy with the fewest number of modes. If the transient responses of the thermal system can be characterized a priori, as is the case in this work due to a structural model created by Witeof and Neergaard, ${ }^{2}$ the method of Proper Orthogonal Decomposition (POD) may be used. ${ }^{22}$ The POD method provides an inherent optimality condition of providing the most efficient capture of the dominant energy modes of a system with a finite number of basis modes ${ }^{23}$ and has been widely used in literature to reduce both linear ${ }^{24,25}$ and nonlinear ${ }^{26}$ thermal $^{2}$ problems.

For the structural dynamics, ROMs are often applications of the Rayleigh-Ritz/Galerkin methods ${ }^{27}$ and matrix transformation to the eigenvalue and eigenvector form. ${ }^{28}$ Once expressed as a set of eigenvectors or modes, the basis set is truncated to reduce the degrees of freedom of the system and thus reduce the effort of integrating the structural equations of motion. However, as the ultimate goal of this work is to capture the coupling of thermal and structural effects, this approach cannot be applied directly to the problem studied here because of the change of the structural stiffness from geometric stiffening and material degradation effects. Instead, the approach taken in this paper was to perform an initial calculation of the free-vibration mode shapes at some reference thermal state. These mode shapes are then to be used as the modal basis for simulation with updates to the stiffness matrix from a kriging surrogate model based on training samples in different thermal states described by the thermal POD basis modes. ${ }^{29}$

\section{Theoretical Development}

\section{A. Aerodynamics Model}

To determine the flow conditions and surface pressures on the vehicle's outer mold line (OML), a combination of normal shock, oblique shock, Prandtl-Meyer expansion, ${ }^{33}$ and piston theory ${ }^{34}$ was used. On any given panel of the OML, the steady component of the surface flow conditions was found using the shock and expansion theories. An unsteady correction was applied to the surface pressure to account for body accelerations, rotations, and structural deformations which caused the panel to move into or out of the flow in its normal direction. This correction is given by the third-order piston theory, that is,

$$
\Delta p=\rho a^{2}\left(M_{n}+\frac{\gamma+1}{4} M_{n}^{2}+\frac{\gamma+1}{12} M_{n}\right)
$$

where $\Delta p$ is the unsteady correction due to a panel's motion expressed as a normal Mach number $M_{n}$. The local flow density $\rho$ and speed of sound $a$ were calculated local to each panel based on the steady shock and expansion solution. The ratio of specific heats $\gamma$ was assumed constant at 1.4. It is important to note that an underlying assumption of piston theory is that the normal motion generates only locally isentropic waves, thus $M_{n}<1$. Corrections to the temperature and Mach number due to unsteadiness may then be considered using the isentropic relations and definition of the Mach number, namely

3

American Institute of Aeronautics and Astronautics

DISTRIBUTION A. Approved for public release, distribution unlimited. (96TW-2015-0158) 


$$
\begin{gathered}
T=T_{0}\left(\frac{p+\Delta p}{p_{0}}\right)^{\frac{\gamma-1}{\gamma}}, \\
M=\frac{v}{\sqrt{\gamma R T}},
\end{gathered}
$$

where $p_{0}$ and $T_{0}$ are the local total pressure and temperature, respectively, $p$ is the steady component of the local static pressure, $v$ is the local steady flow velocity, and $R$ is the gas constant for air, assumed constant at $287 \mathrm{~J} / \mathrm{K} / \mathrm{kg}$.

\section{B. Trajectory Optimization}

In order to tailor thermal and structural modal solutions, a representative terminal trajectory was required. To determine a realistic trajectory, an optimization of the vehicle flight dynamics was carried out to maximize the kinetic energy of the vehicle upon reaching the ground. The General Purpose Optimal Control Software (GPOPSII) ${ }^{12}$ was used with the 3 degree of freedom equations of motion

$$
\begin{gathered}
\dot{r}=v \sin (\beta), \\
\dot{x}_{l o n}=\frac{v \cos (\beta)}{r}, \\
\dot{v}=-\frac{D}{m}-g \sin (\beta), \\
\frac{L}{m}-\cos (\beta)\left(g-\frac{v^{2}}{r}\right) \\
\dot{\beta}=\frac{\frac{m}{v}}{}
\end{gathered}
$$

Here $r$ is the radius from the center of the Earth to the body center, $x_{l o n}$ is the longitude in the Earth frame, $v$ is the speed, $\beta$ is the flight path angle, $g$ is the acceleration due to gravity, $D$ is the drag force, $L$ is the lift force, and $m$ is the vehicle mass. The vehicle was considered to be on a spherical Earth with gravitational acceleration as a function of altitude, lift and drag coefficients derived from the shock, expansion, and piston (SEP) theory aerodynamics model previously described, and atmospheric conditions based on the 1976 standard atmospheric model. ${ }^{35}$ Control was effected by varying the angle of attack which varied the drag $D$ and lift $L$ according to polars derived from the aerodynamic model and the supersonic Prandtl-Glauert correction.

\section{Aeroheating Model}

During the course of the hypersonic trajectory, the outer surface will be exposed to significant aerodynamic heating. To characterize this heating, the Eckert reference temperature method was considered. With the Eckert reference temperature method, variations in the flow properties across a boundary layer are accounted for by a reference temperature which is used to determine integrated properties through the thickness of the boundary layer. The precise profile of the properties cannot be determined with this method, but this approach has been shown to provide surface quantities such as skin friction and heat transfer with acceptable accuracy. ${ }^{36}$

To begin, the static pressure $p$, Mach number $M$, and static temperature $T$ local to each panel of the OML was collected from the SEP aerodynamics model any time the surface heat flux was required. These values were treated as the outer flow conditions of a turbulent boundary layer. A turbulent boundary layer was assumed due to the yet unknown transition characteristics of the flow, and thus provided a worse-case scenario for the heat transfer. This lead to a recovery factor of 


$$
r_{f}=\operatorname{Pr}^{1 / 3},
$$

where the $\operatorname{Pr}$ is the Prandtl number which we have assumed to be constant at 0.7. By definition, the recovery temperature was then

$$
T_{r}=r_{f}\left(T_{0}-T\right)+T,
$$

which allowed Eckert's reference temperature to be found as

$$
T^{*}=T+0.5\left(T_{w}-T\right)+0.22\left(T_{r}-T\right),
$$

where $T_{w}$ is the local wall temperature on the OML. The reference boundary layer flow properties were then

$$
\begin{gathered}
\rho^{*}=\frac{p}{R T^{*}}, \\
\mu^{*}=\mu_{r e f} \frac{T_{r e f}+S}{T^{*}+S}\left(\frac{T^{*}}{T_{r e f}}\right)^{3 / 2}, \\
\operatorname{Re}^{*}=\frac{\rho^{*} v x}{\mu^{*}} .
\end{gathered}
$$

Here the reference density $\rho^{*}$ is found using the ideal gas law. The reference dynamic viscosity $\mu^{*}$ is found using Sutherland's law of viscosity with Sutherland's reference temperature $T_{r e f}=288 \mathrm{~K}$ and Sutherland's constant for air $S=110 \mathrm{~K}$. The reference Reynolds number $\mathrm{Re}^{*}$ is then determined by definition using the local steady flow velocity $v$ outside of the boundary layer and distance along the body from the stagnation point, $x$. The coefficient of skin friction was then determined by the Blasius relation for $\operatorname{Re}^{*} \leq 4.56 \times 10^{6}$,

$$
c_{f}=\frac{0.0592}{\left(\operatorname{Re}^{*}\right)^{0.2}},
$$

and the Schultz-Grunow equation ${ }^{37}$ for $\mathrm{Re}^{*}>4.56 \times 10^{6}$,

$$
c_{f}=\frac{0.37}{\left(\log _{10} \mathrm{Re}^{*}\right)^{2.584}} .
$$

The recommended Reynolds number to transition between the skin friction models is $10^{7}$ by Arthur, Schultz, and Guard ${ }^{37}$ however for the work presented in this paper, the authors adjusted this threshold to have a continuous transition between the models. Both models are necessary since a vehicle 1 to 10 meters in length, traveling at low hypersonic velocities, and stratospheric altitudes will encounter Reynolds numbers on the orders of $10^{6}$ to $10^{8}$. The Stanton number was then determined by the Colburn-Reynolds analogy,

$$
\mathrm{St}=k \frac{c_{f}}{2} \operatorname{Pr}^{-2 / 3}
$$

where $k$ is a geometry dependent coefficient. For a flat plate $k=1$, and for a cone $k=1.28$, as shown by Young and Van Driest. ${ }^{38}$ The heat transfer coefficient can be found from the definition of the Stanton number

American Institute of Aeronautics and Astronautics

DISTRIBUTION A. Approved for public release, distribution unlimited. (96TW-2015-0158) 


$$
h=\mathrm{St} c_{p} \rho^{*} v
$$

where the constant pressure specific heat capacity $c_{p}$ was found using the simple harmonic vibrator model for air

$$
c_{p}=\left(c_{p}\right)_{p e r f}\left\{1+\frac{\gamma-1}{\gamma}\left[\left(\frac{\Theta}{T^{*}}\right)^{2} \frac{\exp \left(\Theta / T^{*}\right)}{\left(\exp \left(\Theta / T^{*}\right)-1\right)^{2}}\right]\right\}
$$

where $\left(c_{p}\right)_{\text {perf }}=1006 \mathrm{~J} / \mathrm{K} / \mathrm{kg}$ is the constant pressure specific heat capacity of calorically perfect air and $\Theta=2778 \mathrm{~K}^{\text {perf }}$ s the characteristic temperature of the diatomic vibrational mode of air. Finally, the heat flux to the surface is given by

$$
\dot{q}=h\left(T_{r}-T_{w}\right)+\sigma \varepsilon\left(T_{\infty}^{4}-T_{w}^{4}\right)
$$

where $\sigma=5.6704 \times 10^{-8} \mathrm{~W} / \mathrm{m}^{2} / \mathrm{K}^{4}$ is the Stefan-Boltzmann constant, $\varepsilon$ is the emissivity of the surface material, and $T_{\infty}$ is the far-field freestream temperature from the 1976 standard atmospheric model, ${ }^{35}$ thus accounting for the conductive and radiative heat transfer.

\section{Thermal Model Reduction}

To reduce the order of the thermal model, we have followed the work of Falkiewicz and Cesnik ${ }^{22}$ and sought to express the temperature distribution of the vehicle structure as the sum of a small number of basis vectors multiplied by time varying coefficients, i.e.,

$$
\left\{\begin{array}{c}
T_{1} \\
\vdots \\
T_{s}
\end{array}\right\}=c_{1}(t)\left\{\begin{array}{c}
\phi_{1}^{(1)} \\
\vdots \\
\phi_{s}^{(1)}
\end{array}\right\}+c_{2}(t)\left\{\begin{array}{c}
\phi_{1}^{(2)} \\
\vdots \\
\phi_{s}^{(2)}
\end{array}\right\}+\ldots+c_{r}(t)\left\{\begin{array}{c}
\phi_{1}^{(r)} \\
\vdots \\
\phi_{s}^{(r)}
\end{array}\right\},
$$

where $T_{i}$ is the temperature of the $i$ th of $s$ nodes in the structure model, $c_{j}(t)$ is the $j$ th of $r$ time-varying coefficients of the thermal basis vectors, and $\phi_{i}^{(j)}$ is the $i$ th entry of the $j$ th thermal basis vector. To determine the thermal basis vectors $\phi$, the method of snapshots is used ${ }^{3}$. With the method of snapshots, a high-fidelity thermal model was simulated using finite element analysis (FEA) software. Given a surface heat flux derived from the aerodynamic and aeroheating models, a transient heat transfer solver was used to simulate the thermal state forward in time. Snapshots of the temperature of each node in the finite element model (FEM) were taken at prescribed time intervals and used to form the snapshot matrix

$$
\mathbf{A}=\left[\begin{array}{cccc}
T_{1}^{(1)} & T_{1}^{(2)} & \cdots & T_{1}^{(n)} \\
T_{2}^{(1)} & T_{2}^{(2)} & \cdots & T_{1}^{(n)} \\
\vdots & \vdots & \ddots & \vdots \\
T_{s}^{(1)} & T_{s}^{(2)} & \cdots & T_{s}^{(n)}
\end{array}\right]
$$

where each column is a vector of the FEM nodal temperatures at a single moment in the simulation, $T_{i}^{(j)}$ is the $i$ th of $s$ node temperatures at the time the $j$ th of $n$ snapshots of the thermal state. A correlation matrix $\mathbf{C}$, was then formed from the snapshot matrix $\mathbf{A}$ as

$$
\mathbf{C}=\frac{1}{n} \mathbf{A}^{T} \mathbf{A}
$$

The eigenvectors and eigenvalues of the covariance matrix $\mathbf{C}$ are then found from

American Institute of Aeronautics and Astronautics

DISTRIBUTION A. Approved for public release, distribution unlimited. (96TW-2015-0158) 


$$
\mathbf{C v}=\lambda v,
$$

where $\boldsymbol{v}$ is a matrix whose columns are the eigenvectors corresponding to the entries of the diagonal matrix $\boldsymbol{\lambda}$ which contains the eigenvalues of $\mathbf{C}$ arranged in decreasing magnitude. The thermal basis vectors were then determined as

$$
\phi_{k}=\frac{1}{\sqrt{n \lambda_{k}}} \mathbf{A} \mathbf{v}_{k}
$$

where $\underline{\phi}_{k}$ is the $k$ th thermal basis vector, $n$ is the number of snapshots originally taken, $\underline{\lambda}_{k}$ is the $k$ th diagonal entry of the eigenvalue matrix, and $\mathbf{v}_{k}$ is the $k$ th column of the eigenvector matrix. Arranging the base thermal modes as the columns of a matrix gives the thermal basis matrix

$$
\boldsymbol{\Phi}=\left[\begin{array}{ccc}
\phi_{1}^{(1)} & \cdots & \phi_{1}^{(n)} \\
\vdots & \ddots & \vdots \\
\phi_{s}^{(1)} & \cdots & \phi_{s}^{(n)}
\end{array}\right]=\left[\phi_{1}, \ldots, \phi_{n}\right]
$$

where $\boldsymbol{\Phi}$ is the thermal basis matrix whose columns are the thermal basis vectors. Note that $n$ thermal basis vectors are determined since $n$ thermal snapshots were considered by the snapshot matrix. With this thermal basis matrix, the snapshot matrix may be reproduced exactly as ${ }^{24}$

$$
\mathbf{A}=\boldsymbol{\Phi} \boldsymbol{\eta}
$$

where $\eta$ is an $n$-dimensional square matrix of coefficients of the thermal basis vectors $\phi_{k}$ which, when summed, reproduce the thermal state at that time. Thus, row $k$ of $\eta$ is a time history of the magnitude of basis $\underline{\phi}_{k}$ during simulation at each of the original snapshots.

To reduce the order of the thermal basis matrix, it was considered that $\lambda_{1}$ was the largest magnitude eigenvalue and thus $\underline{\phi}_{1}$ contains the most dominant thermal basis. Correspondingly, $\lambda_{n}$ was the smallest magnitude eigenvalue and $\phi_{n}$ contains the least dominant thermal basis. Truncating the thermal basis matrix by removing the least dominant thermal modes allows one to reduce the number of thermal degrees of freedom while preserving the most dominant features of the thermal state,

$$
\boldsymbol{\Psi}=\left[\begin{array}{ccc}
\phi_{1}^{(1)} & \cdots & \phi_{1}^{(m)} \\
\vdots & \ddots & \vdots \\
\phi_{s}^{(1)} & \cdots & \phi_{s}^{(m)}
\end{array}\right]=\left[\phi_{1}, \ldots, \phi_{m}\right] ; m<n
$$

where $\boldsymbol{\Psi}$ is the truncated thermal basis matrix. By truncating the thermal basis, one loses the ability to exactly reproduce the snapshot matrix. However, the snapshot matrix can still be approximated by

$$
\mathbf{A} \approx \mathbf{\Psi c},
$$

where $\mathbf{c}$ is now an $m$ by $n$ matrix of the coefficients of the truncated thermal basis vectors. The error incurred by truncation of the thermal basis matrix may be interpreted as the relative energy lost $\varepsilon_{\text {rel }}$ by projecting the snapshot matrix $\mathbf{A}$ of $n$ dimension onto the $m$ dimensional space spanned by the truncated thermal basis matrix $\boldsymbol{\Psi}$, given by $^{39}$

$$
\varepsilon_{r e l}=\frac{\left\|\mathbf{A}-\boldsymbol{\Psi} \boldsymbol{\Psi}^{T} \mathbf{A}\right\|^{2}}{\|\mathbf{A}\|^{2}} .
$$




\section{E. Structural Model Reduction}

To reduce the order of the structural model, the structural equations of motion can be generalized by a set of orthogonal free-vibration mode shapes. To determine the mode shapes, the Abaqus FEM/CAE ${ }^{30}$ linear perturbation, frequency domain, Lanczos ${ }^{32}$ solver was used to determine

$$
\left(-\omega_{j}^{2} \mathbf{M}+\mathbf{K}\right) \boldsymbol{\xi}_{j}=0
$$

where $\omega_{j}$ is the frequency (square-root of the eigenvalue) of the $j$ th free-vibration mode, $\mathbf{M}$ is the assembled mass matrix, $\mathbf{K}$ is the assembled stiffness matrix, and $\xi_{j}$ is the $j$ th free-vibration mode shape vector. The mode shapes are normalized by mass, and therefore satisfy

$$
\begin{gathered}
\mathbf{I}=\boldsymbol{\xi}^{T} \mathbf{M} \boldsymbol{\xi}, \\
{\left[\omega^{2}\right]=\boldsymbol{\xi}^{T} \mathbf{K} \boldsymbol{\xi},}
\end{gathered}
$$

where $\mathbf{I}$ is the identity matrix and $\xi$ is the mode shape matrix whose columns are the mode shape column vectors $\xi_{j}$ corresponding to $\left[\omega^{2}\right]$ which is a diagonal matrix whose entries are the squares of the free-vibration frequencies.

\section{Representative Vehicle Model}

\section{A. Vehicle Configuration}

The methodology described in this work is applied to a representative hypersonic vehicle configuration. The configuration was largely established by Witeof and Neergaard ${ }^{2}$ using the PASS code suite ${ }^{1}$ and is shown in Figure 2, Figure 3, and Figure 4. Basic properties are given in Table 1 and component materials in Table 2.
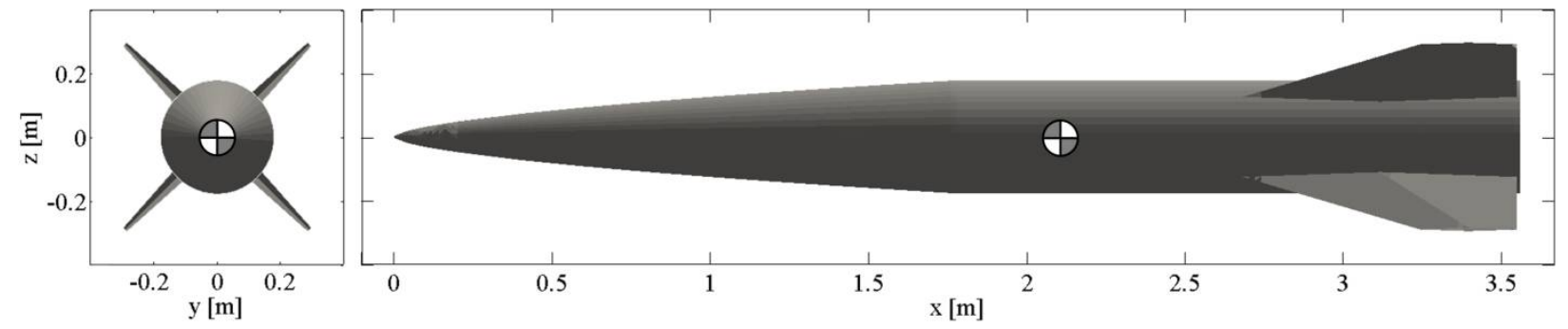

Figure 2: Representative vehicle dimensions

Table 1: Basic properties

\begin{tabular}{lcc} 
Property & $\begin{array}{c}\text { Fully } \\
\text { Fueled }\end{array}$ & $\begin{array}{c}\text { Reserve } \\
\text { Fuel Only }\end{array}$ \\
\hline Body length $(\mathrm{m})$ & 3.56 & 3.56 \\
Body diameter $(\mathrm{m})$ & 0.36 & 0.36 \\
Wingspan $(\mathrm{m})$ & 0.82 & 0.82 \\
Center of gravity (m) & $2.09,0,0$ & $2.06,0,0$ \\
Mass $(\mathrm{kg})$ & 375 & 307 \\
$\mathrm{I}_{\mathrm{xx}}\left(\right.$ about CG) $\left(\mathrm{kg} \mathrm{m}^{2}\right)$ & 9.42 & 7.37 \\
$\mathrm{I}_{\mathrm{yy}}\left(\right.$ about CG) $\left(\mathrm{kg} \mathrm{m}^{2}\right)$ & 345 & 338 \\
$\mathrm{I}_{\mathrm{zz}}$ (about CG) $\left(\mathrm{kg} \mathrm{m}^{2}\right)$ & 345 & 338
\end{tabular}

The body is axisymmetric with four aft all movable fins for stability and control. Beginning at the nose tip, the OML starts with a 10-mm diameter hemisphere tangent-transitioned to power-law forebody following the relation:

8

American Institute of Aeronautics and Astronautics

DISTRIBUTION A. Approved for public release, distribution unlimited. (96TW-2015-0158) 


$$
r=0.126 x^{0.6},
$$

where $r$ is the radius in meters of the OML $x$ meters along the axis of symmetry from the nose. At $1.78 \mathrm{~m}$ from the nose, the body transitions to a uniform cylinder $0.36 \mathrm{~m}$ in diameter, which continues until the trailing end, 3.56 $\mathrm{m}$ from the nose tip. The fins, shown in Figure 3, are diamond airfoils with a maximum thickness at 50\% chord. The maximum thickness is $5 \%$ of the chord, plus a $1 \mathrm{~mm}$ TPS layer. Internally, the fins are solid QISO $^{\mathrm{TM}}$ triaxial weave carbon-carbon composite which is assumed to be quasi-isotropic. The root chord is $86.4 \mathrm{~cm}$ and tapers down to a $30.4 \mathrm{~cm}$ chord at the tip. The leading edge is swept back $67.3^{\circ}$ and the trailing edge is unswept. The fins are attached to the body by $17.8 \mathrm{~cm}$ long, $2.0 \mathrm{~cm}$ diameter solid shafts, $51.8 \mathrm{~cm}$ from the leading edge and formed from the same carbon-carbon composite as the internal fin structure. The center of each fin shaft is attached to the body $3.2 \mathrm{~m}$ from the nose tip.

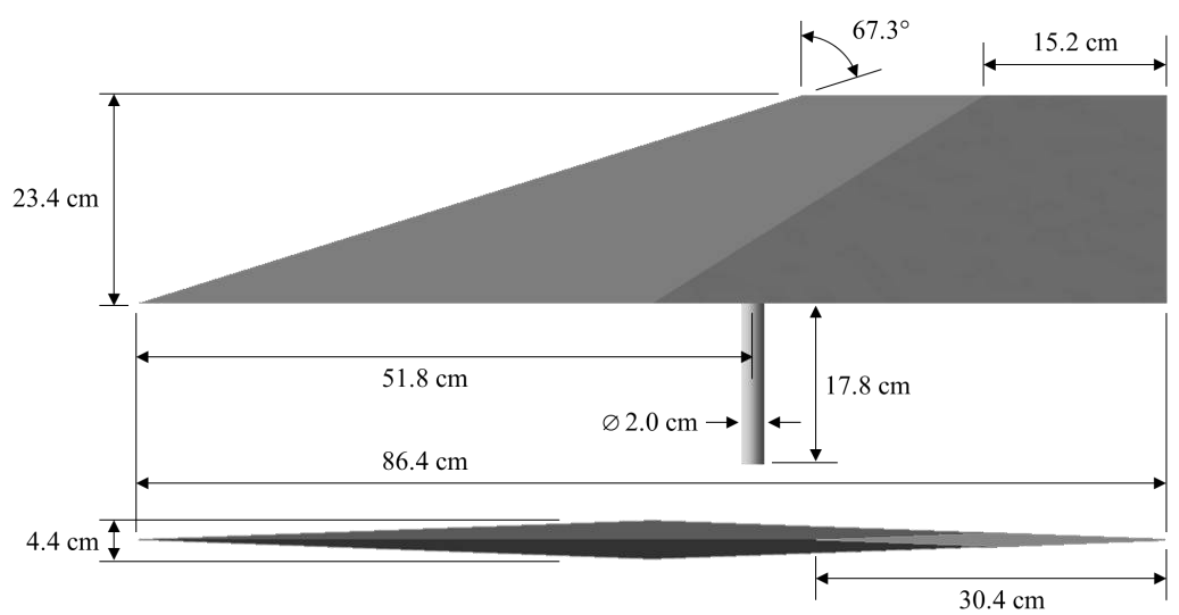

Figure 3: Fin OML dimensions

Shown in Figure 4, the internal structure is a titanium alloy monocoque with additional stiffeners between a fin root box and trailing end. The purpose of the stiffeners is to support the body during the boost phase of flight when a rocket motor mounted to the trailing end is used to bring the vehicle to cruising speed. The thickness of the monocoque skin varies between $1.3 \mathrm{~mm}$ and $3.1 \mathrm{~mm}$ according to an optimization performed previously using the PASS code suite. ${ }^{1}$ Covering the monocoque skin is a TPS of Exelis ${ }^{\mathrm{TM}}$ Acusil-II ${ }^{40}$ material. Like the monocoque skin, the TPS varies in thickness according to optimization results from the PASS code suite, ${ }^{1}$ but generally is thicker near the nose, thins along the body toward the tail, and thickens again aft of the fin root shafts to effect a cooler, stiffer structure near the fin root box. At the nose is a solid tungsten ballast which forms the first $20 \mathrm{~cm}$ of the nose which bears the stagnation heat flux during hypersonic flight and brings the center of gravity forward. Within the body is also a $90 \mathrm{~kg}$ steel casing which acts as a thermal sink for temperature sensitive components. During analysis, the steel casing is considered only as a thermal sink and its mass is not directly used. Instead, nonstructural masses are distributed along the monocoque skin to account for various internal subsystems.

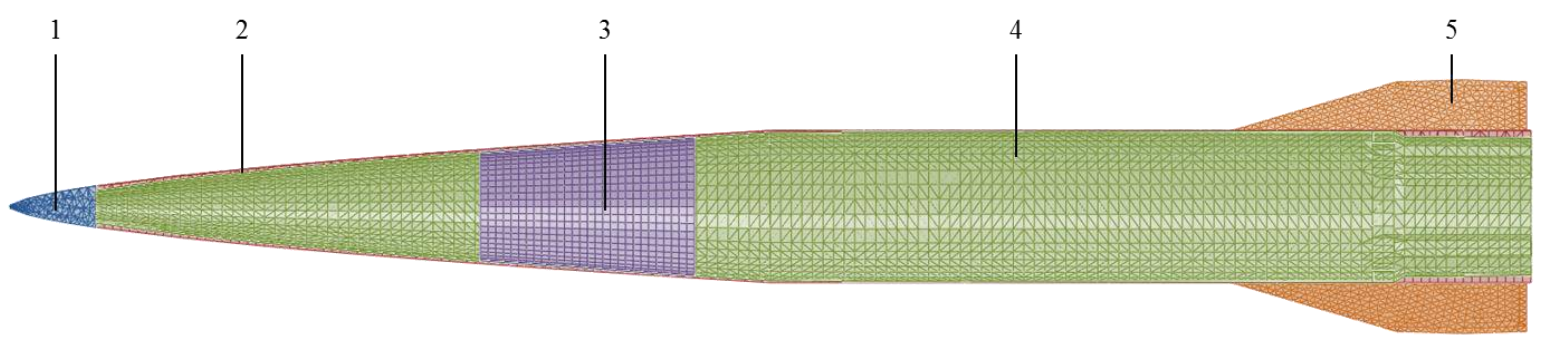

Figure 4: Representative vehicle internal structure cross-section and FEM

9

American Institute of Aeronautics and Astronautics

DISTRIBUTION A. Approved for public release, distribution unlimited. (96TW-2015-0158) 
Table 2: Representative vehicle materials (see Figure 4)

\begin{tabular}{cll} 
ID & Component & Material(s) \\
\hline 1 & Nose ballast & Tungsten \\
2 & Thermal protection system & Acusil-II \\
3 & Casing & Carbon steel \\
4 & Monocoque & Titanium alloy \\
5 & Control fins & QISO carbon-carbon composite \& Acusil-II
\end{tabular}

\section{B. Material Properties}

It is expected that the operating temperatures will be sufficient to cause significant changes to the properties of its constituent materials. Thus data for material properties over a wide range of temperatures were required and given in Table 3, and Figure 5. The TPS material, Acusil-II, is assumed to not contribute to the structural stiffness and was assigned a Young's modulus of $0.01 \mathrm{GPa}$, so to be low but not cause numerical instability. Material properties were found in Ref. 40 through 47.

\section{Finite Element Model}

To provide a reference and training sample source for the ROM creation, a pair of FEMs were created. The first was a structural model for vibration mode analysis and load testing. The second was a thermal model for heat transfer analysis. Both models are based on the work of Witeof and Neegard, ${ }^{2}$ however mesh refinement and other adjustments have been made to the previous work to permit thermal analyses pertaining to both FEMs. A summary of the node count, element count, and element types used in each components of the FEM is given in Table 4 and Table 5. In both models, thick components such as the ballast and body TPS, were modeled with solid elements. Thin-walled components, such as the monocoque, steel casing, fin structures, and fin TPS were modeled with shell elements. The fin root shafts joining the fins to the body were modeled as cubic beam elements. For the thermal FEM, heat transfer between the fins and the body was assumed negligible and the fin root shafts were neglected. For the structural FEM, the mass contribution of the steel casing was already accounted for by the distributed nonstructural masses added to the monocoque skin, thus the casing mesh was unnecessary. The element and component layout is shown in Figure 4.

\section{Results and Discussion}

\section{A. Aerodynamics model}

The OML was extracted from the structural and thermal FEMs and used to determine the surface pressures for flight at Mach 6.5 and $65,000 \mathrm{ft}$ altitude for various angles of attack. The surface pressure distributions were then compared to Euler CFD simulations processed by the FUN3D code suite by Dreyer. ${ }^{48}$ The SEP and CFD solutions for surface pressure are shown in Figure 6.

It can be seen that overall the SEP solutions qualitatively match well with the Euler CFD solutions for surface pressure. However there are minor differences including the over-prediction of pressure at the nose tip by the SEP model due to the neglect of 3D pressure relief effects for conical bodies. Also, the shock wave interaction between the fins and the body is neglected since the SEP model considers panels of the OML individually, with no account for the regions of dependence or influence in the supersonic flow. That said, calculation of each SEP pressure distribution required $\sim 6$ seconds on 1 Intel E5 $2.0 \mathrm{GHz}$ core while calculation of each Euler CFD solution required $\sim 300$ seconds on 128 Intel E5 $2.6 \mathrm{GHz}$ cores.

Quantitatively, we have compared the pressure solution at the centroid of each panel of the SEP solution with the spatially nearest panel of the CFD solution. The normalized root-mean-square error (NRMSE) is used,

$$
\mathrm{NRMSE}=\frac{\sqrt{\left[\frac{1}{n} \sum_{i=1}^{n}\left(p_{S E P, i}-p_{C F D, i}\right)\right]^{2}}}{\max \left(p_{C F D}\right)-\min \left(p_{C F D}\right)},
$$


where $n=21315$ is the number of panels comprising the SEP OML, $p_{S E P, i}$ is the surface pressure at the centroid of the $i$ th panel of the SEP OML, and $p_{C F D, i}$ is the surface pressure at the spatially nearest CFD panel to the SEP panel. The NRMSE for each angle of attack is given in Table 6.

Table 3: Temperature independent material properties

\begin{tabular}{lrc} 
& $\begin{array}{r}\text { Density } \\
{\left[\mathrm{kg} / \mathrm{m}^{3}\right]}\end{array}$ & $\begin{array}{c}\text { Poisson's } \\
\text { Ratio }\end{array}$ \\
\hline Acusil-II & 256.3 & 0.30 \\
CC-QISO & 1650.0 & 0.21 \\
Carbon Steel & 7900.0 & 0.30 \\
Ti-6Al-4V & 5199.4 & 0.31 \\
Tungsten & 19250.0 & 0.26
\end{tabular}
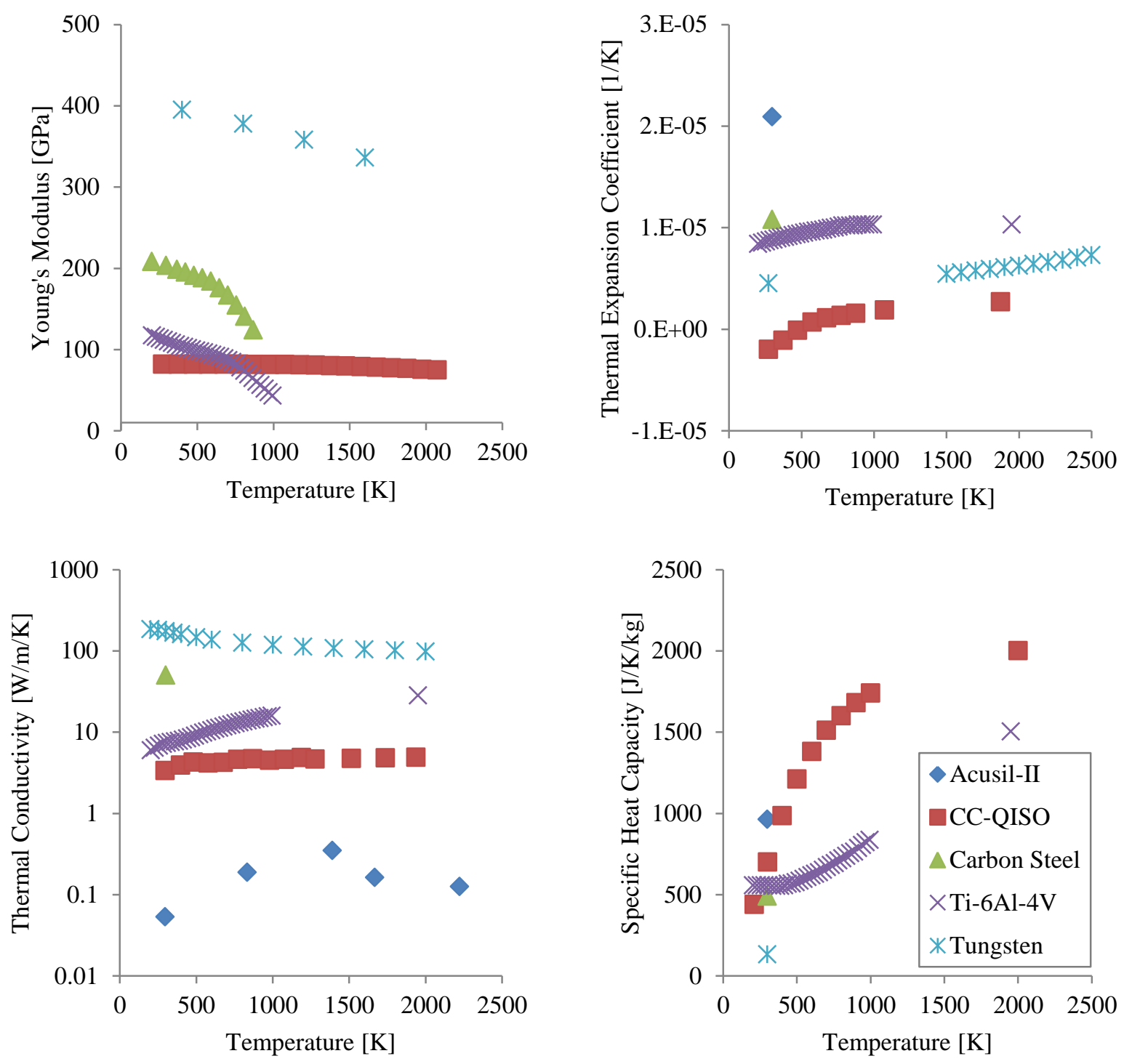

Figure 5: Temperature dependent material properties, Refs. 40-47

11

American Institute of Aeronautics and Astronautics

DISTRIBUTION A. Approved for public release, distribution unlimited. (96TW-2015-0158) 


\begin{tabular}{lrrll} 
& \multicolumn{2}{c}{ Table 4: Structural Abaqus FEM mesh details } & \\
Node & $\begin{array}{l}\text { Element } \\
\text { count }\end{array}$ & Element type & $\begin{array}{l}\text { Element } \\
\text { code }\end{array}$ \\
\hline Ballast & 2177 & 1279 & quadratic tetrahedral & C3D10 \\
Monocoque & 5509 & 11096 & linear triangular & S3R \\
TPS & 14620 & 7267 & linear hexahedral & C3D8 \\
Fin & 1300 & 3 & cubic beam & B33 \\
& & 2371 & linear triangular & S3R
\end{tabular}

Table 5: Thermal Abaqus FEM mesh details

\begin{tabular}{|c|c|c|c|c|}
\hline Component & $\begin{array}{l}\text { Node } \\
\text { count }\end{array}$ & $\begin{array}{l}\text { Element } \\
\text { count }\end{array}$ & Element type & $\begin{array}{l}\text { Element } \\
\text { code }\end{array}$ \\
\hline Ballast & 2375 & 1403 & quadratic tetrahedral & DC3D10 \\
\hline Monocoque & 5509 & 11096 & linear triangular & DS3 \\
\hline TPS & 14620 & 7267 & linear hexahedral & DC3D8 \\
\hline Casing & 1479 & 1428 & linear quadrilateral & DS4 \\
\hline Fin & 983 & 1795 & linear triangular & DS3 \\
\hline
\end{tabular}

Shock, Expansion, Piston Theory

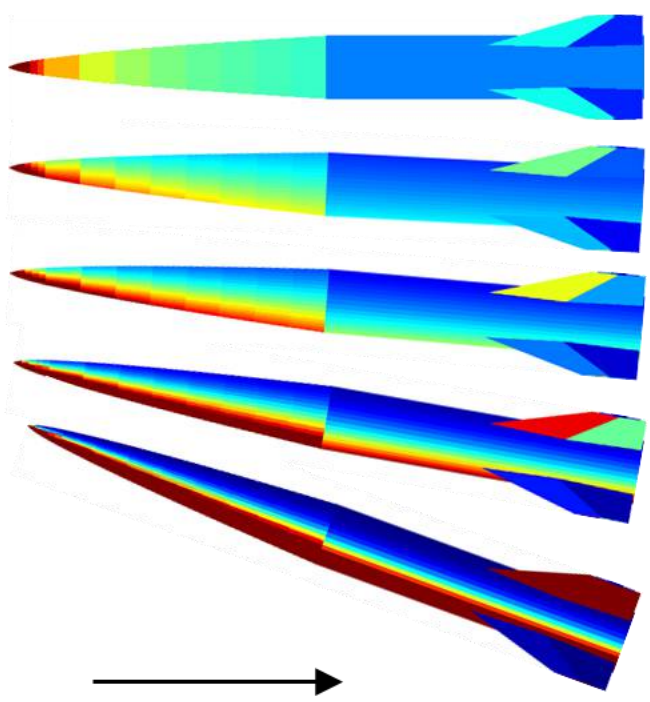

Direction of flow $M=6.5$

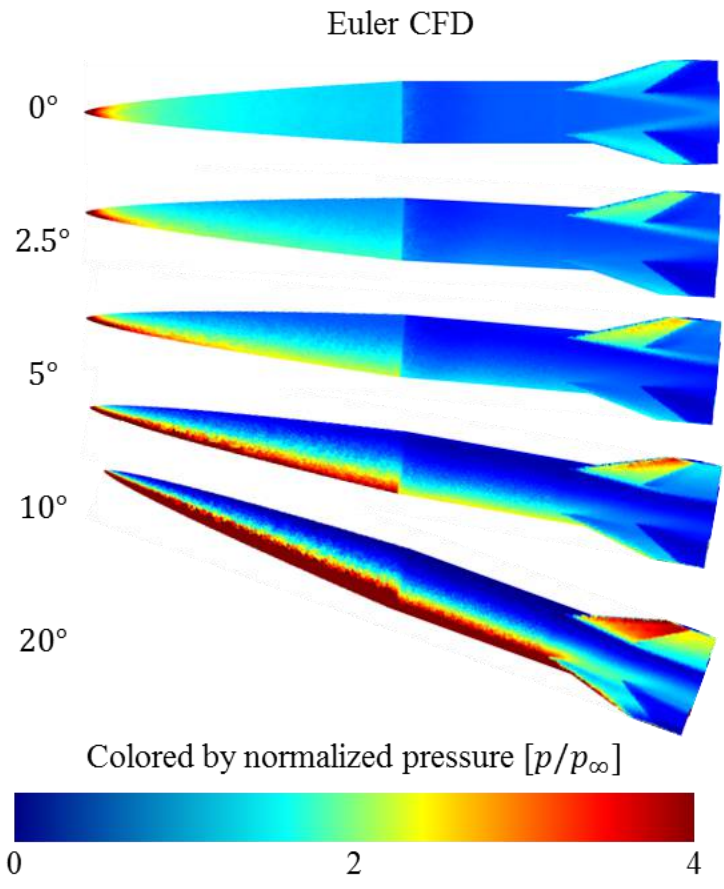

Figure 6: Comparison of SEP to Euler CFD surface pressures

Table 6: Comparison of the SEP and CFD pressure solutions

\begin{tabular}{cc} 
Angle of attack [deg] & NRMSE \\
\hline 0.0 & $1.28 \%$ \\
2.5 & $1.31 \%$ \\
5.0 & $1.45 \%$ \\
10.0 & $2.05 \%$ \\
20.0 & $4.39 \%$
\end{tabular}

12

American Institute of Aeronautics and Astronautics

DISTRIBUTION A. Approved for public release, distribution unlimited. (96TW-2015-0158) 


\section{B. Vehicle Trim}

While analysis of the terminal phase trajectory was the primary goal, the thermal initial conditions to the terminal phase are established by the cruise phase. Thus, trimming for steady-level flight in the cruise was required to determine the flow conditions over the OML required to determine the surface heat flux. During the cruise phase, an undescribed propulsion system is active to maintain flight. For the purposes of this paper, the authors have assumed that this propulsion system consumes all non-reserve fuel linearly over the duration of a 500-nautical-mile cruise phase. This cruise phase is assumed to be greater than Mach 5, and above $50 \mathrm{kft}(15.2 \mathrm{~km})$ in altitude. Trimming was conducted by minimizing the summed magnitudes of the body forces and moments for a number of time instances along the cruise phase using the simplex method implemented through the fminsearch built-in function of Matlab. ${ }^{31}$ Inputs to the minimization were the angle of attack and fin deflection. The fin deflection was applied to each fin symmetrically across the plane of symmetry for longitudinal flight to reduce all fin deflections to a single angle. A thrust force was applied as an axial force along the body axis of symmetry and was scaled to cancel the component of the weight along the axis of the vehicle plus the drag in the body frame after the aerodynamic model was applied,

$$
T=D+m g \cos (\alpha),
$$

where $T$ is the thrust magnitude, $m$ is the mass of the vehicle, $g$ is the acceleration due to gravity, $\alpha$ is the angle of attack, and $D$ is the drag, taken to be the $x$ component of the net aerodynamic forces in the body frame obtained from the SEP aerodynamics model. Note that the SEP aerodynamic model used in this work was akin to an Euler solution in that it ignored viscous effects and accounted only for pressure drag. To ensure that the neglect of the viscous drag would not significantly affect the trim solution, a set of trim solutions including the skin friction coefficients derived from the Eckert reference temperature method were considered for a uniform OML temperature of $273 \mathrm{~K}$. While the required thrust increased, sometimes by as much as $38 \%$, the angle of attack and fin deflection angles varied by less than $4 \%$ compared to the inviscid trim solution. Since the incident angle of the OML to the freestream flow is the primary driving factor for the surface heat flux considered later in this paper, the inviscid trim solutions were considered adequate. The aerodynamic forces in the body frame are found by

$$
\mathbf{F}=-\left[\begin{array}{ccc}
p_{1} A_{1} \hat{n}_{x}^{(1)} & p_{1} A_{1} \hat{n}_{y}^{(1)} & p_{1} A_{1} \hat{n}_{z}^{(1)} \\
\vdots & \vdots & \vdots \\
p_{n} A_{n} \hat{n}_{x}^{(n)} & p_{n} A_{n} \hat{n}_{y}^{(n)} & p_{n} A_{n} \hat{n}_{z}^{(n)}
\end{array}\right]
$$

and aerodynamic moments in the body frame are found by

$$
\mathbf{M}=\left[\mathbf{x}_{c}\right]_{\times} \mathbf{F},
$$

where $\mathbf{F}$ is a $n \times 3$ matrix of forces on each OML panel, $p_{i}$ is the pressure on the $i$ th OML panel determined from the SEP aerodynamics model, $A_{i}$ is the surface area of the ith OML panel, $\hat{n}_{x}^{(i)}, \hat{n}_{y}{ }^{(i)}, \hat{n}_{z}^{(i)}$ are the components of the outward facing unit normal vector of the $i$ th OML panel, $\mathbf{M}$ is a $n \times 3$ matrix of moments about the nose tip, $\mathbf{x}_{c}$ is a $n \times 3$ matrix of the body frame locations of the OML panel centroids from the nose, and [ $]$ is the skewsymmetric matrix operator. Total drag $D$ and lift $L$ are the sums of the first and third columns of the force matrix $\mathbf{F}$, respectively. Total pitching moment is the sum of the second columns of the moment matrix. The net forces and moments relevant for steady-level flight, assuming lateral symmetry, are then

$$
\begin{gathered}
F_{x, \text { net }}=\sum_{i=1}^{n} F_{\mathrm{i}, 1}+m g \sin (\alpha)-T, \\
F_{\mathrm{z}, \text { net }}=\sum_{i=1}^{n} F_{\mathrm{i}, 3}+m g \cos (\alpha),
\end{gathered}
$$

13

American Institute of Aeronautics and Astronautics

DISTRIBUTION A. Approved for public release, distribution unlimited. (96TW-2015-0158) 


$$
M_{\mathrm{y}, \text { net }}=\sum_{i=1}^{n} M_{\mathrm{i}, 2}+x_{C G} m g \cos (\alpha)
$$

where $F_{i, j}$ is the $i$,jth entry of $\mathbf{F}, M_{i, j}$ is the $i, j$ th entry of $\mathbf{M}$, and $x_{C G}$ is the distance of the center of gravity from the nose tip. Note that eq. (38) is identically 0 under all conditions due to the definition of the thrust $T$ in eq. (35). Thus only $F_{z . n e t}$ and $M_{y, n e t}$ must be minimized to achieve obtain the trim state. Since at this point, the elastic and thermal models were not yet developed and the structure was considered perfectly rigid. Shown in Figure 7 is a sample lift and drag distribution along the body length for the end of the cruise phase at Mach 6 and an altitude of $75 \mathrm{kft}(22.9 \mathrm{~km})$. Figure 8 shows the trim conditions for the beginning and end of the cruise phase at the flight conditions described.

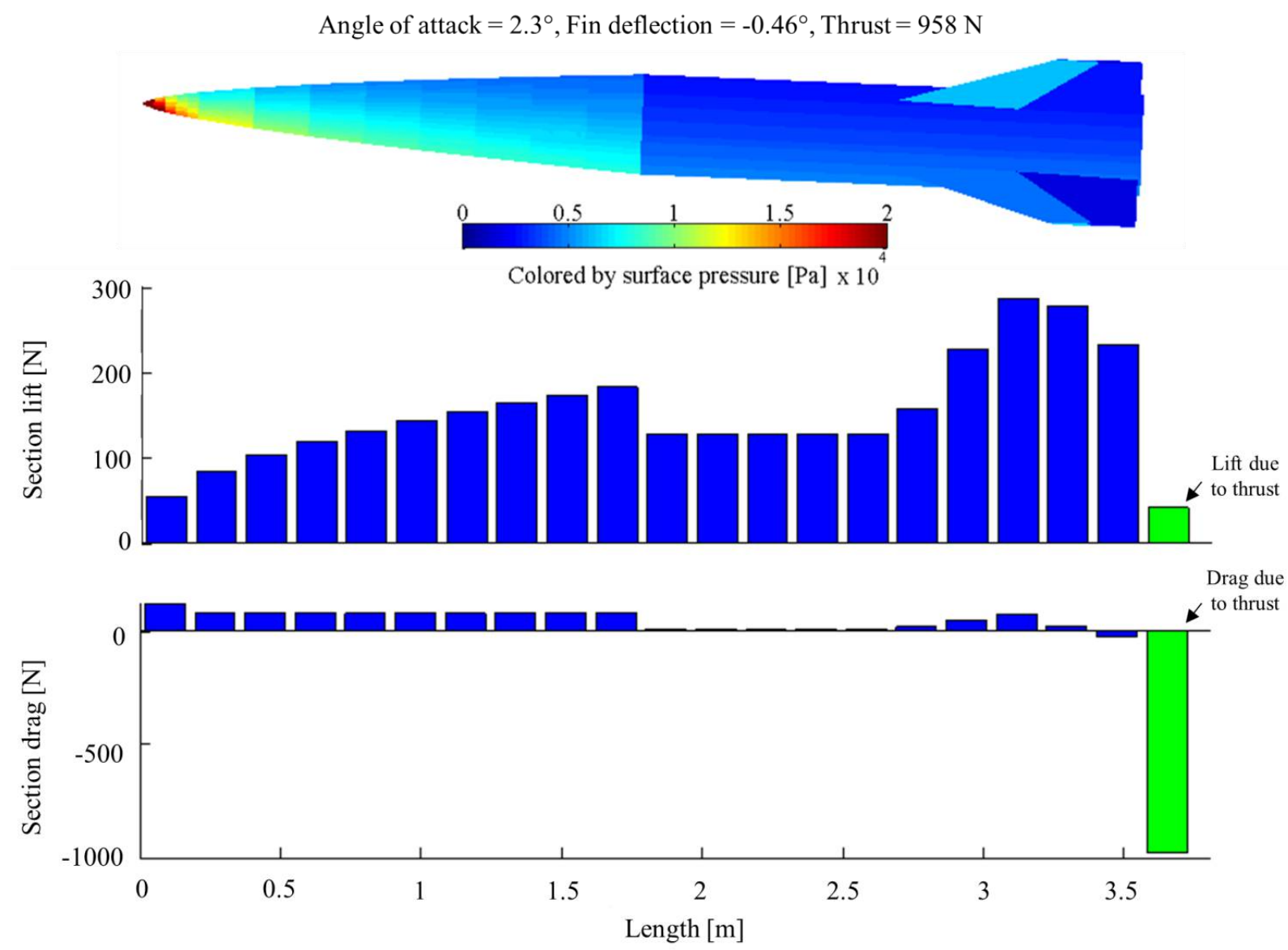

Figure 7: Sample lift and drag distribution for Mach 6, $75 \mathrm{kft}(22.9 \mathrm{~km})$ altitude, end of cruise phase

\section{Terminal Trajectory Optimization}

The vehicle enters the terminal phase of the trajectory following the cruise phase. In the terminal phase, the vehicle pitches downward and begins its descent toward the ground. To this end, the GPOPS-II code was used to determine optimal terminal trajectories which maximized the final kinetic energy.

To integrate the previously described equations of motion (4) through (7), lift and drag polars were required. The SEP aerodynamics model was used to vary the angle of attack at Mach 6.5, and record the variation of the coefficients of lift and drag. The results are shown in Figure 9.

14

American Institute of Aeronautics and Astronautics

DISTRIBUTION A. Approved for public release, distribution unlimited. (96TW-2015-0158) 

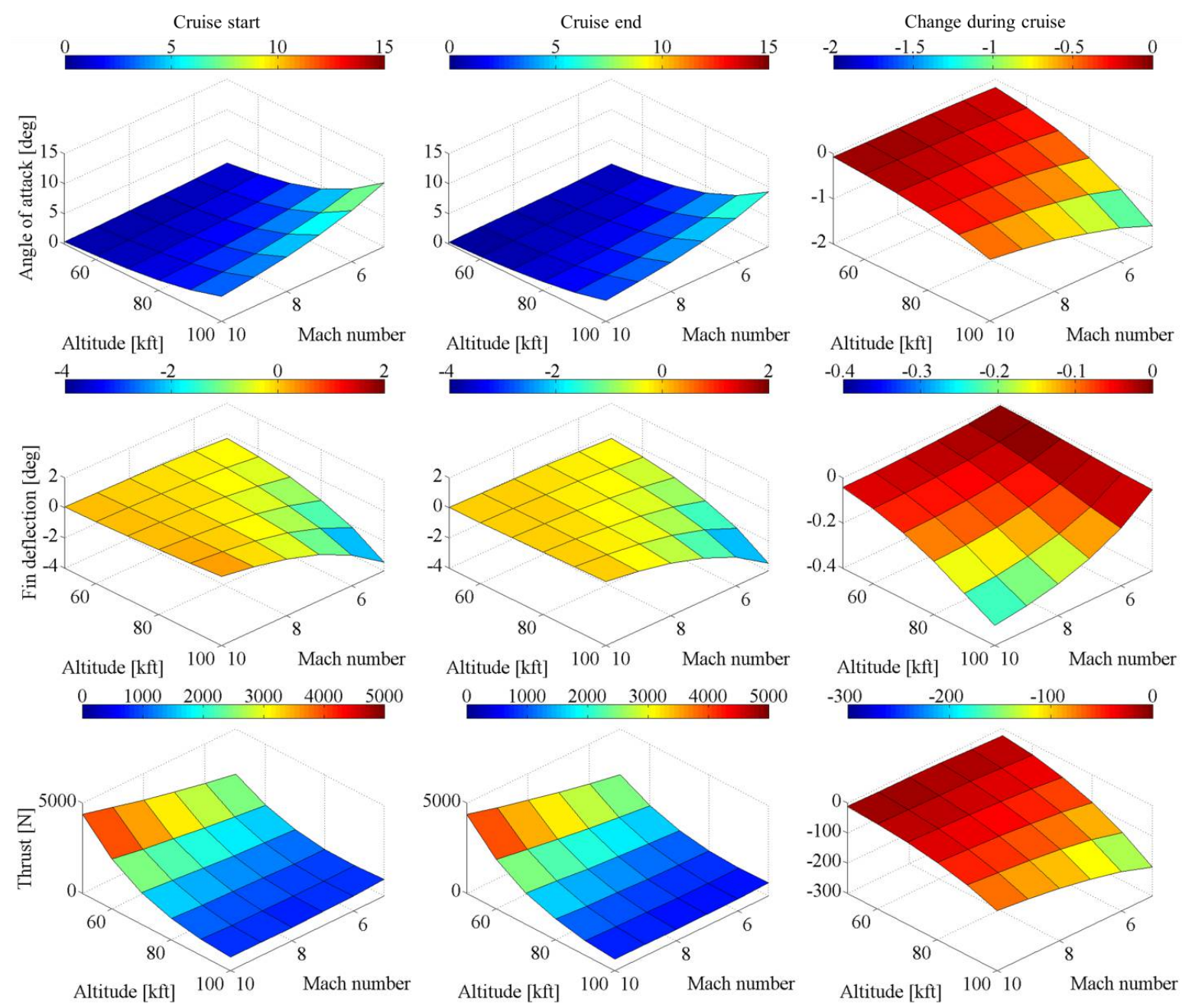

Figure 8: Cruise phase trim states for a range of Mach numbers and altitudes 

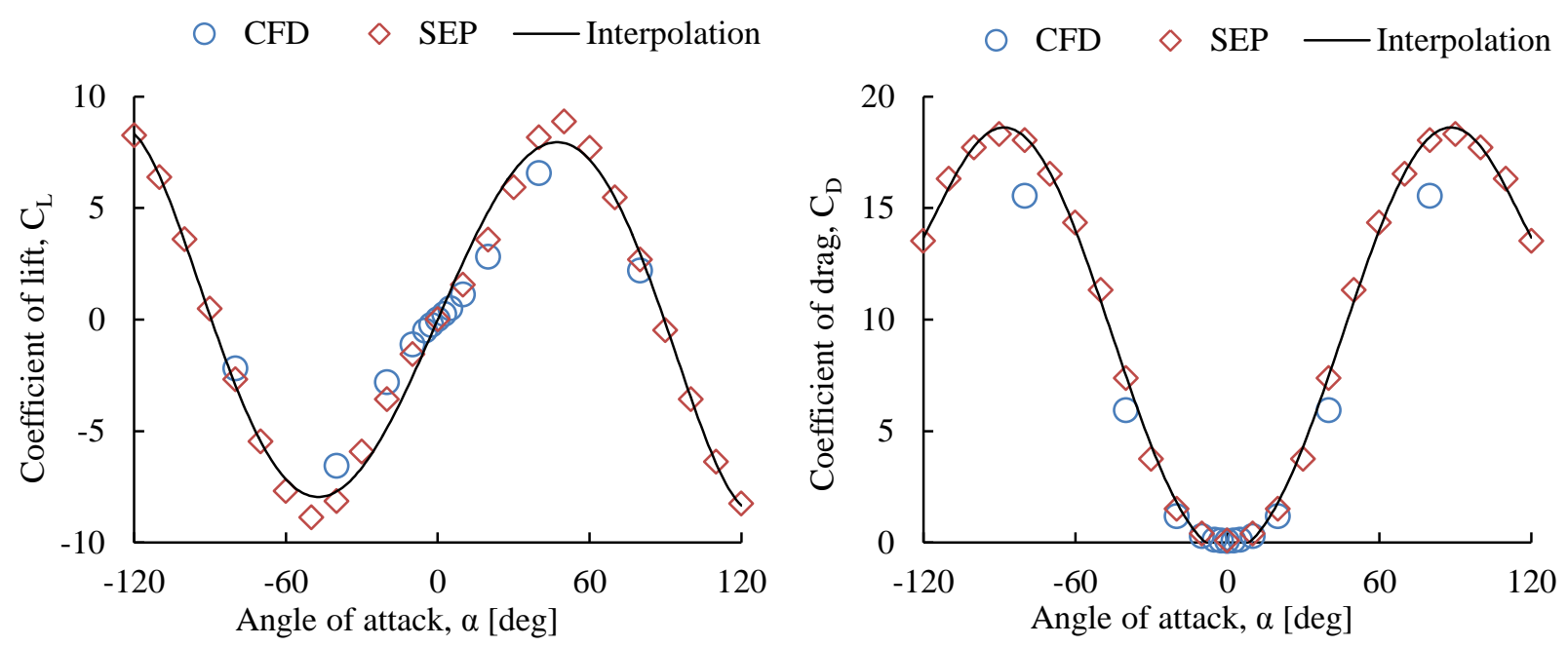

Figure 9: Vehicle lift and drag polars for Mach 6.5

A sparse sampling of CFD solutions taken from Ref. 36 are also shown in Figure 9 for comparison to the SEP solutions. Overall, both solutions match well for both moderate and extreme angles of attack pre- and post-stall. The least-squares method was then used to fit fifth and sixth order polynomials to each polar, yielding

$$
\begin{gathered}
C_{L}(M=6.5)=\left(2.603 \times 10^{-1}\right) \alpha^{5}+\left(-4.456 \times 10^{-5}\right) \alpha^{3}+\left(1.504 \times 10^{-9}\right) \alpha, \\
C_{D}(M=6.5)=\left(8.478 \times 10^{-2}\right) \alpha^{6}+\left(5.450 \times 10^{-3}\right) \alpha^{4}+\left(-4.908 \times 10^{-7}\right) \alpha^{2}+\left(3.482 \times 10^{-2}\right),
\end{gathered}
$$

where $C_{L}$ is the coefficient of lift, $C_{D}$ is the coefficient of drag, and $M$ is the Mach number. The Prandtl-Glauert factor for supersonic flight was then used to approximate $C_{L}$ and $C_{D}$ for other Mach numbers

$$
\begin{gathered}
C_{L}(M)=\frac{C_{L}(M=6.5) \sqrt{(6.5)^{2}-1}}{\sqrt{M^{2}-1}}, \\
C_{D}(M)=1.5 \times \frac{C_{D}(M=6.5) \sqrt{(6.5)^{2}-1}}{\sqrt{M^{2}-1}} .
\end{gathered}
$$

A factor of 1.5 was also applied to $C_{D}$ to account for additional drag generated by an undescribed scramjet inlet. Given $C_{L}, C_{D}$, and the equations of motion, the terminal trajectories shown in Figure 10 for initial Mach numbers $5,6,7,8,9$, and 10 and initial altitudes of 50,60,70, 80, 90, and $100 \mathrm{kft}(15.2,18.3,21.3,24.4,27.4$, and $30.5 \mathrm{~km}$, respectively) were generated by the GPOPS-II code to maximize final kinetic energy.

American Institute of Aeronautics and Astronautics

DISTRIBUTION A. Approved for public release, distribution unlimited. (96TW-2015-0158) 


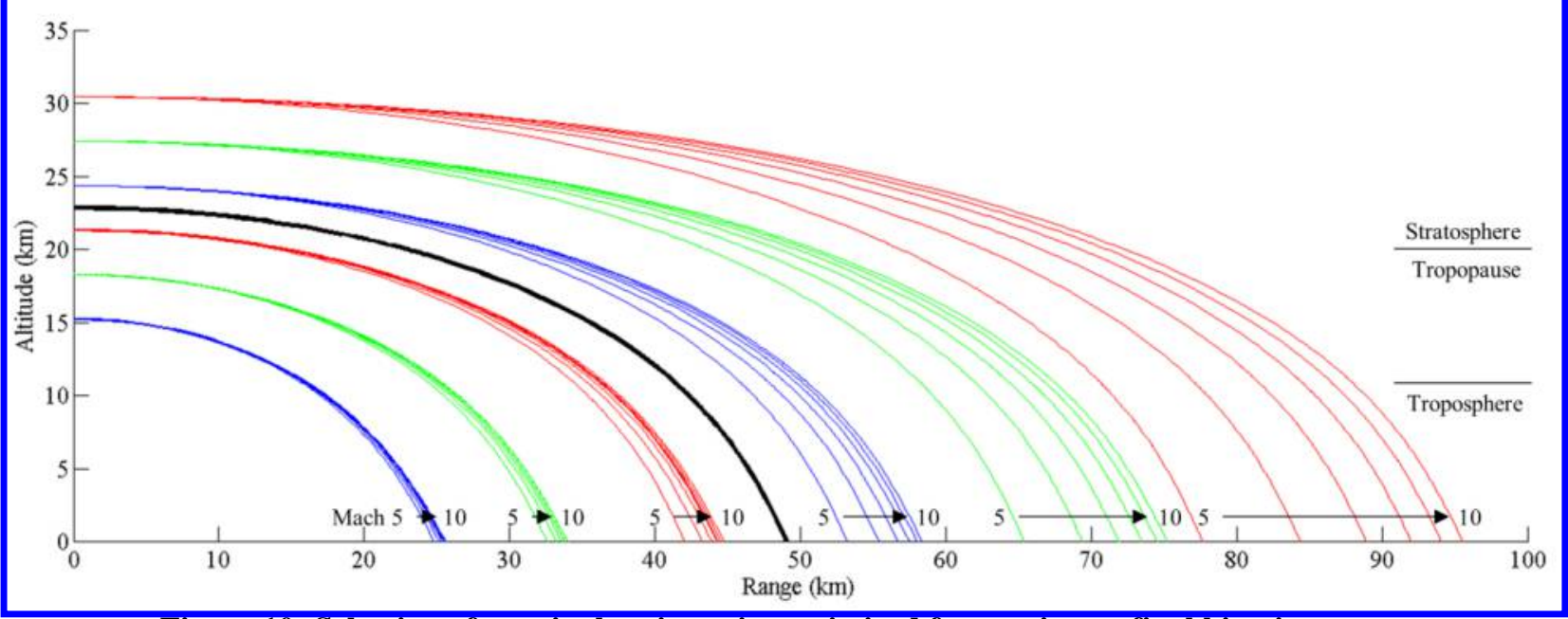

Figure 10: Selection of terminal trajectories optimized for maximum final kinetic energy

A representative trajectory starting at Mach $6,75 \mathrm{kft}(22.9 \mathrm{~km})$ altitude was selected for further analysis. The trajectory is overlaid and bolded onto Figure 10 for comparison to the sample set of trajectories. The time histories of the altitude, flight speed, angle of attack, and flight path angle of the representative trajectory are shown in Figure 11 .

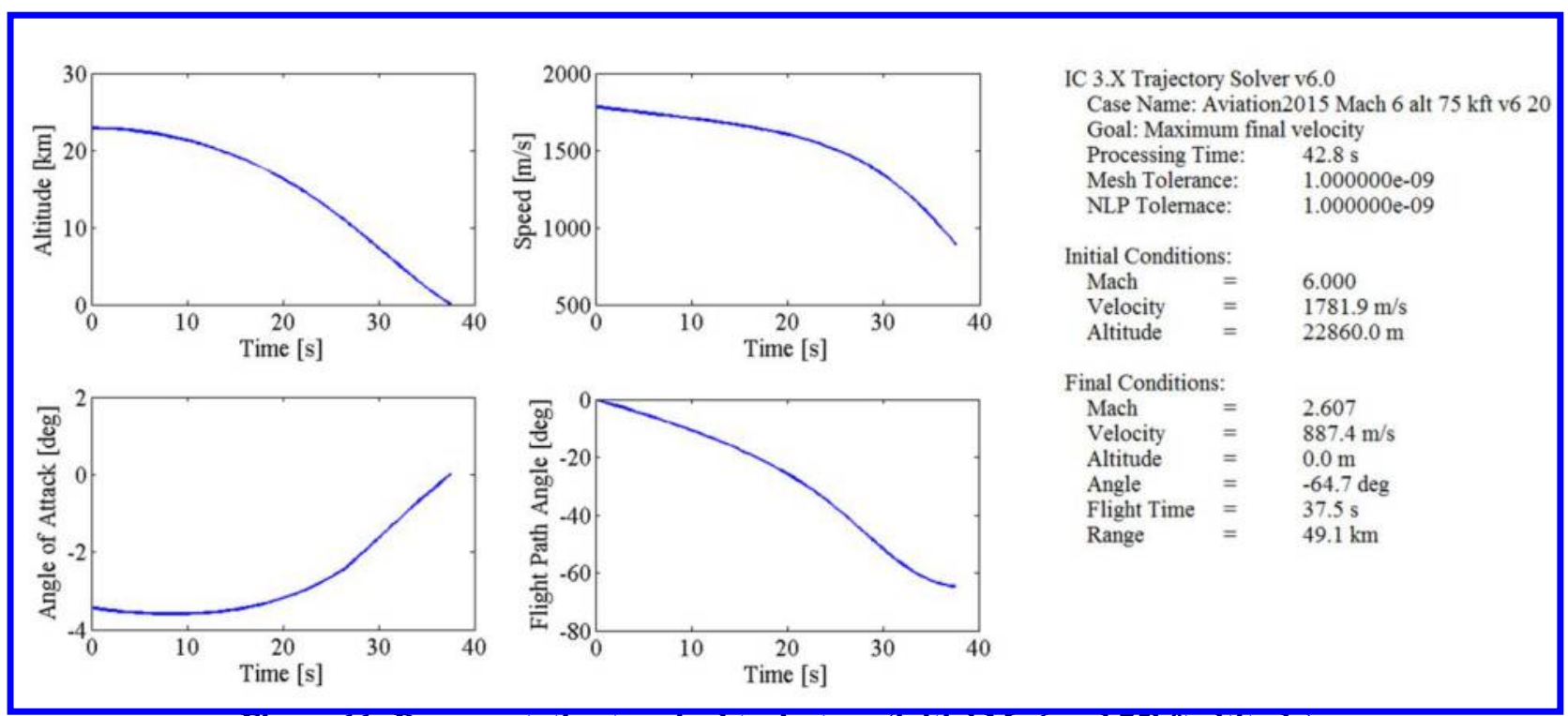

Figure 11: Representative terminal trajectory (initial $M=6$ and $75 \mathrm{kft}$ altitude)

The total flight time of the representative terminal trajectory is $37.5 \mathrm{~s}$, covering a range of $49.1 \mathrm{~km}$. The final Mach number is 2.6 , equivalent to $887 \mathrm{~m} / \mathrm{s}$.

\section{Thermal Model Reduction}

To employ the method of snap shots to the thermal state in the terminal trajectory phase, a high-fidelity heat-transfer simulation of the structure was required. However, simply simulating the terminal phase is insufficient due to the thermal hysteresis. The cruise phase was also simulated to determine the initial terminal phase heat transfer conditions.

An Abaqus user-defined subroutine (UDS) was written to interface the heat-transfer FEA with the SEP aerodynamic model and trim solver. Within the UDS, the Eckert aeroheating model was implemented and used to determine the surface heat flux across the aerodynamic boundary layer given flow conditions determined a priori and wall temperatures determined during the heat-transfer FEA. The process flow chart is shown in Figure 12.

17

American Institute of Aeronautics and Astronautics

DISTRIBUTION A. Approved for public release, distribution unlimited. (96TW-2015-0158) 


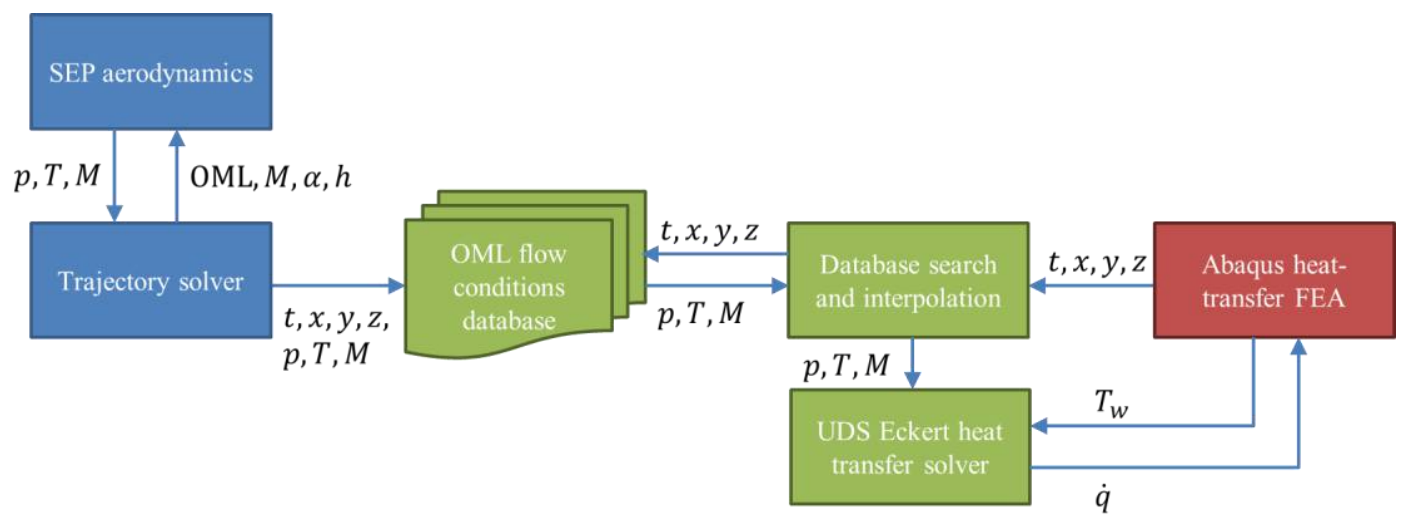

Figure 12: Process for coupling aerodynamic model and structure heat-transfer FEA

During preprocessing, the trajectory solver, which during cruise is the trim solver, passes the OML geometry, flight Mach number, angle of attack, and altitude to the SEP aerodynamics model. The aerodynamics model then returns pressure, outer temperature, and outer Mach number at the centroid of each OML panel. Upon determining the vehicle is trimmed for a given instant in the cruise phase, time, spatial coordinates, pressure, outer temperature, and outer Mach number for each panel are written to a database. During the heat-transfer FEA, the time and spatial coordinates of a node on the FEM are passed by Abaqus to the database searcher and interpolator. Since the aerodynamic solutions are considered at the OML panel centroids and the heat-transfer simulation temperature solutions are considered at the element nodes, interpolation of the flow properties is implemented by averaging the flow solutions of all spatially adjacent panel centroids to an element node, i.e.,

$$
\{p, T, M\}_{\text {node }}=\frac{1}{n} \sum_{i=1}^{n}\{p, T, M\}_{\text {centroid }},
$$

where $n$ is dependent on the element type of the associated node. For quadrilateral elements, $n=4$, while for tetrahedral and triangular elements, $n=6$. No interpolation was considered temporally. Instead, trim and aerodynamic solutions were determined at intervals of 0.1 -s and flow conditions were taken at the nearest recorded time in the OML flow conditions database. This approach was viable since a time interval of 0.1-s was much too fine of an interval to appear in the heat-transfer simulation due to the long characteristic times of thermodynamic processes.

A Mach 6, $75 \mathrm{kft}(22.9 \mathrm{~km})$ altitude cruise heat-transfer simulation was begun with the structure at a uniform 238 $\mathrm{K}$. The cruise phase covered 500 nautical miles $(926 \mathrm{~km})$ over approximately $520 \mathrm{~s}$. Temperature profiles for the OML and bond-line between the monocoque skin, fin structure, and TPS are shown in Figure 13. Maximum and minimum temperatures of each component during simulation are shown in Figure 14. To increase the processing rate of the heat-transfer simulation, the body and fins were simulated separately. Since the fins have less thermal capacity and a larger relative surface area than the body, they tend to experience rapid heating initially, requiring fine time steps, but quickly stabilize and can be integrated with larger time steps. On the other hand, the body does not heat as rapidly, allowing for larger time steps than the fins initially, but spends a longer time in a transient state, thus not allowing the time steps to be lengthened as significantly as with the fins. This separation of the body and fins implies the neglect of gap heating at the joint between the fins and body. While gap heating is a significant source of heat flux, a model for it has not been developed in this work.

18

American Institute of Aeronautics and Astronautics

DISTRIBUTION A. Approved for public release, distribution unlimited. (96TW-2015-0158) 


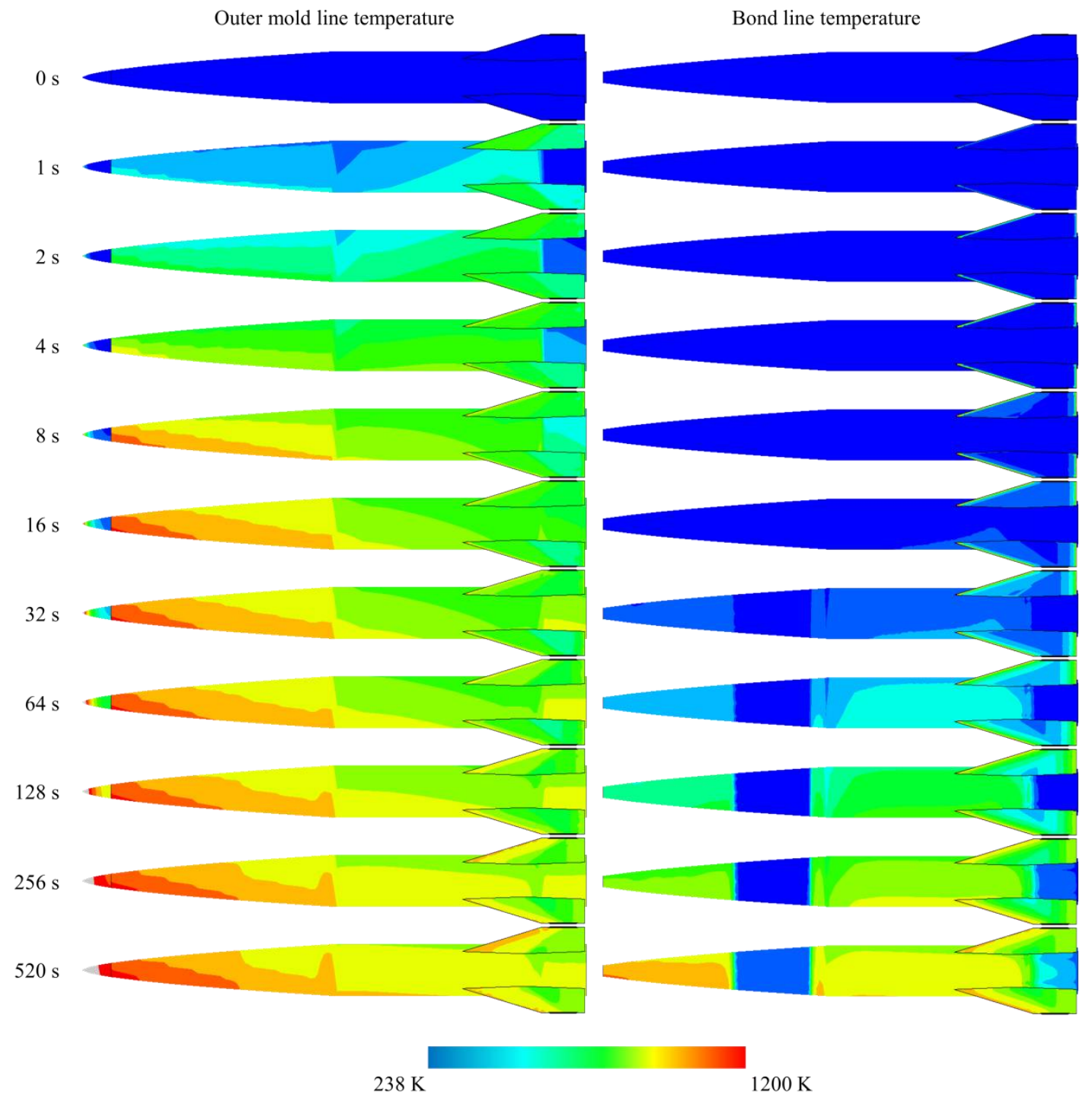

Figure 13: Cruise phase temperature profiles for Mach 6, $75 \mathrm{kft}(22.9 \mathrm{~km})$ altitude

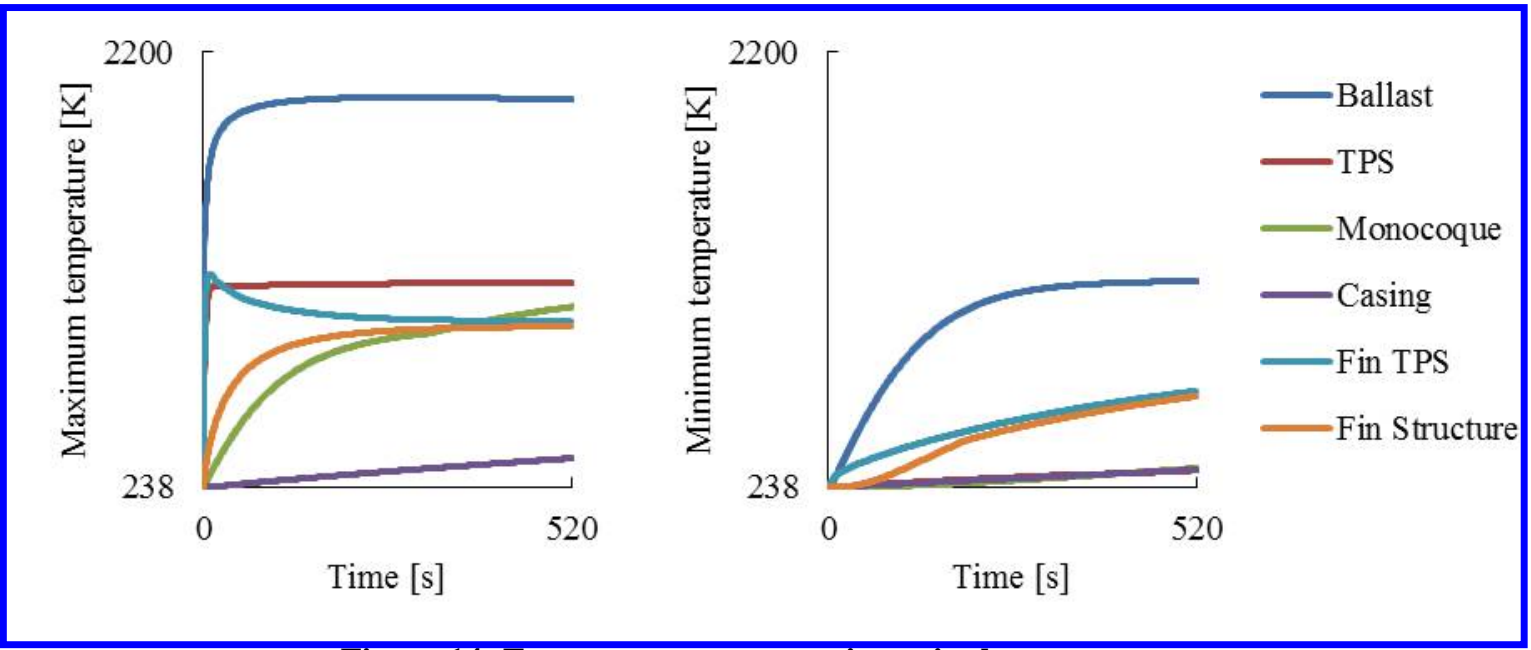

Figure 14: Extreme temperatures in cruise by component

American Institute of Aeronautics and Astronautics

DISTRIBUTION A. Approved for public release, distribution unlimited. (96TW-2015-0158) 
During the cruise phase, the nose and fore-body experience the greatest heating due to the more inclined OML surfaces. This was expected as the TPS is thicker in these areas than the mid-body. The ballast bears the greatest heating due to the stagnation point, but heats relatively slowly compared to the TPS slightly aft due to its greater thermal capacity. One may see that the mid-body monocoque bound line is the first to experience the heating effects at approximately $16 \mathrm{~s}$ into the cruise phase, where the TPS is the thinnest, but ultimately the highest bound line temperature was found on the windward fore-body, despite the thicker TPS on the fore-body. The fin TPS heated nearly as quickly as the stagnation point on the ballast, but quickly reached a maximum and then began to cool slightly as the fins assumed new angles of attack to trim the vehicle.

Following the cruise phase heat-transfer simulation is terminal phase heat-transfer simulation with initial thermal states determined by the final states of the cruise phase. Linking between the SEP aerodynamics model and the Abaqus FEA was nearly identical to what was done for the cruise phase, except that rather than the trajectory being determined by the trim solver, the GPOPS-II optimized terminal trajectory states are used. Transition from the trim condition at the end of cruise to the condition at the beginning of the terminal phase is considered to be instantaneous. In reality, the pitch-over maneuver from the cruise to terminal phase would require some finite amount of time, but the duration of this maneuver was assumed to be small compared to the characteristic time of the thermal solution. As with the cruise phase, aerodynamic solutions in the terminal phase were considered at intervals of 0.1-s and written to the OML flow conditions database and the fin and body heat-transfer simulations were run separately. Temperature profiles for the OML and bond-line between the monocoque skin, fin structure, and TPS are shown in Figure 15 and temperature ranges by component are shown in Figure 16.

The heating effects of the pitch-over maneuver at the initiation of the terminal phase can be seen in the inversion of the maximum heating location on the fore-body OML in the first $4 \mathrm{~s}$. The OML then continues to heat until about $24 \mathrm{~s}$ when the vehicle enters the troposphere and the combination of lower Mach number and higher density atmosphere begins to quench its surface. The duration of the terminal phase appears to be insufficient to significantly alter the thermal state of the monocoque bond line while the fin bound line did experience some cooling near the leading and trailing edges due to the locally low heat capacity of the fin structure. Several sharp "kinks" in the extreme temperature plots of Figure 16 may be seen. These are the result of the extreme temperature point changing to a new location in the structure which experienced a different cooling rate than the previous location, resulting in a discontinuity in the slope of the plotted results.

Throughout both the cruise and terminal phases, one can see the effects of the relative thermal capacities of different components, primarily the ballast and steel casing. The ballast bears the greatest surface heat fluxes, both in heating and cooling, but tended to change overall temperature slowly. The steel casing is protected from the surface heating and remains relatively cool. The lower temperature of the structure in the vicinity of the casing even extends through the monocoque and TPS and is visible in the OML temperature distributions.

With the thermal states known for the terminal phase, the method of snapshots is then applied. Since the ultimate goal is to truncate the resulting modal basis to reduce the number of thermal degrees of freedom, selecting modes which contain the most system energy is desirable. It has been previously shown by Falkiewicz and Cesnik ${ }^{22}$ that, in some cases, taking more snapshots to derive the modal bases can result in a less accurate thermal model due to the spreading of energy to higher modes which are then neglected during truncation. Ideally, enough thermal modes are retained to achieve machine accuracy or roughly 15 significant figures. To determine the accuracy of a truncated mode set, Eq. (29) is used. If it is found that the relative energy loss is approximately 15 orders of magnitude less that the total system energy $\left(\varepsilon_{r e l} \approx 10^{-15}\right)$, then one could consider the energy loss by truncation of the modal basis to be negligible. Thermal snapshots were considered at several time intervals ranging from $10 \mathrm{~s}$ to 0.1 -s. The relative energy loss was then determined for truncation of each modal basis compared to the snapshot matrix used to form them. The number of retained modes required to achieve negligible energy loss given the number of snapshots taken is shown in Figure 17. Once the number of bases retained from each snapshot matrix was determined, the relative energy loss was investigated further by projecting the 0.1-s time interval snapshot matrix onto the space spanned by the retained bases and reevaluating the relative energy loss of the each basis set. The results are shown in Figure 18.

It can be seen from Figure 17 that taking more snapshots indeed results in more bases required to capture the target system energy, which indicates a spreading of energy to higher modes. ${ }^{22}$ However, this spread of energy appears to asymptote to approximately 30 to 35 modes for large numbers of snapshots. From Figure 18, one can see that the relative energy loss decreased steadily for larger numbers of snapshots, which eventually reached the target of $\varepsilon_{\text {rel }} \approx 10^{-15}$ for snapshots taken at 0.1-s intervals. Thus, it was determined that the 33 basis modes derived from the 376 snapshots taken at 0.1 -s intervals provided an adequate representation of the thermal solution in the terminal phase. The first 10 thermal basis modes are shown in Figure 19 with the eigenvalue magnitudes of each mode and relative energy loss $\varepsilon_{\text {rel }}$ due to truncation error shown in Figure 20 and Figure 21. 


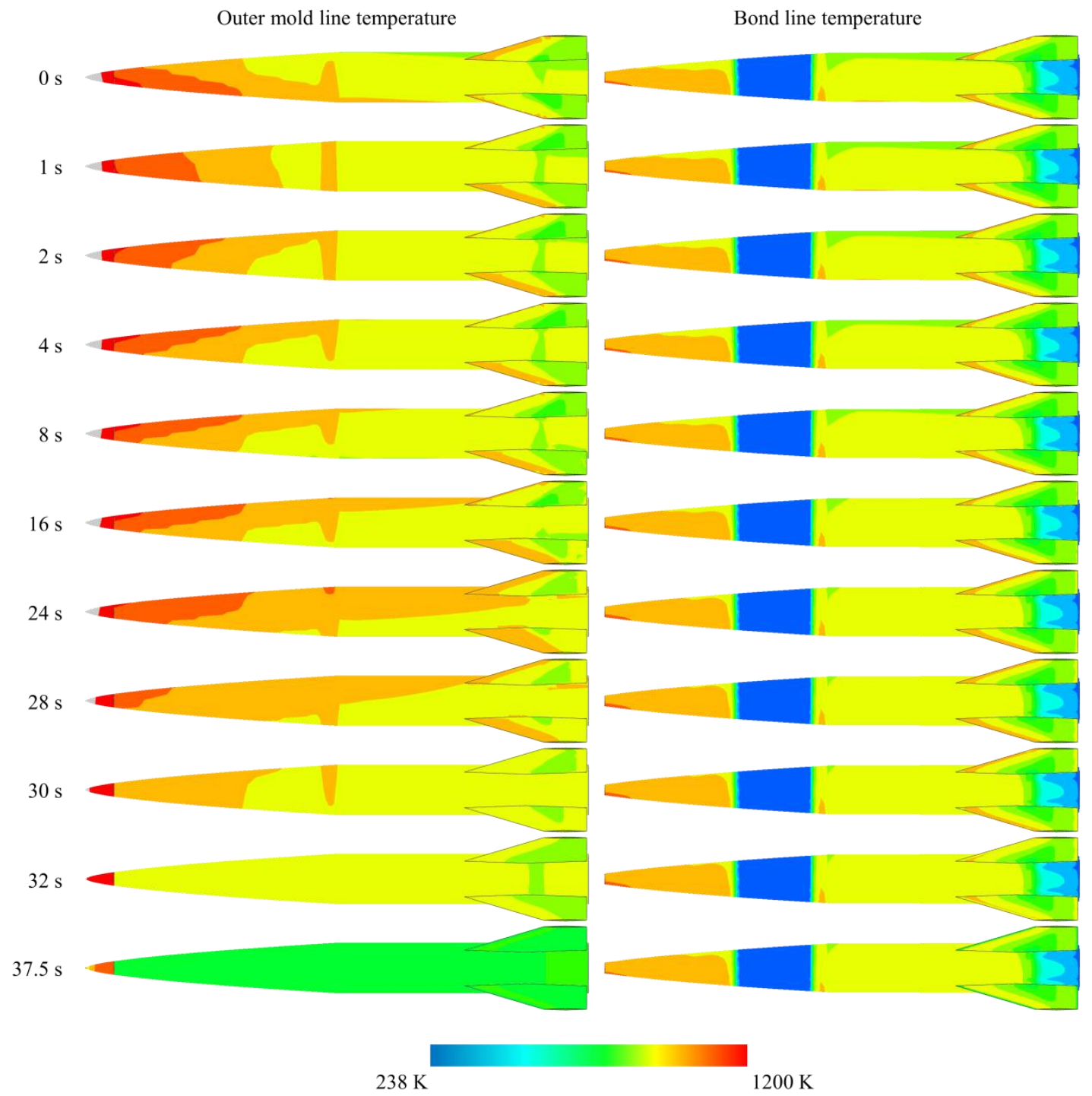

Figure 15: Terminal phase temperature profiles for Mach 6, $75 \mathrm{kft}(22.9 \mathrm{~km})$ altitude
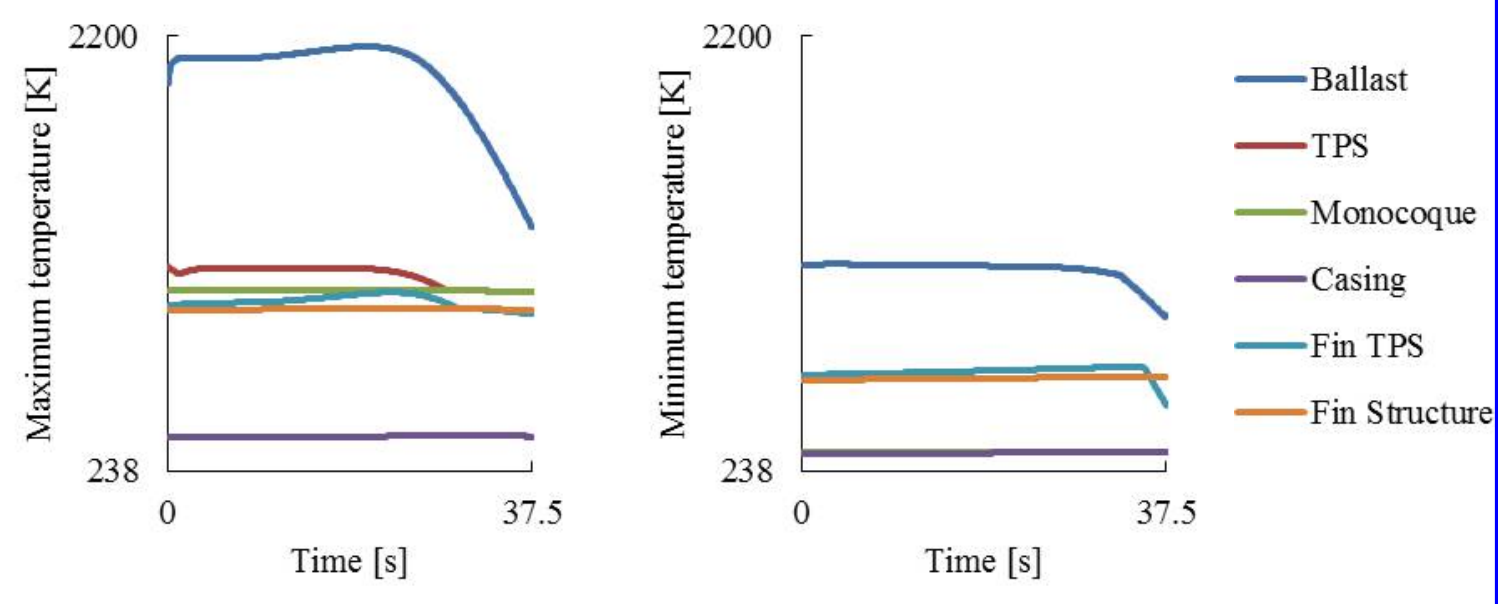

Figure 16: Extreme temperatures in terminal phase by component

American Institute of Aeronautics and Astronautics

DISTRIBUTION A. Approved for public release, distribution unlimited. (96TW-2015-0158) 


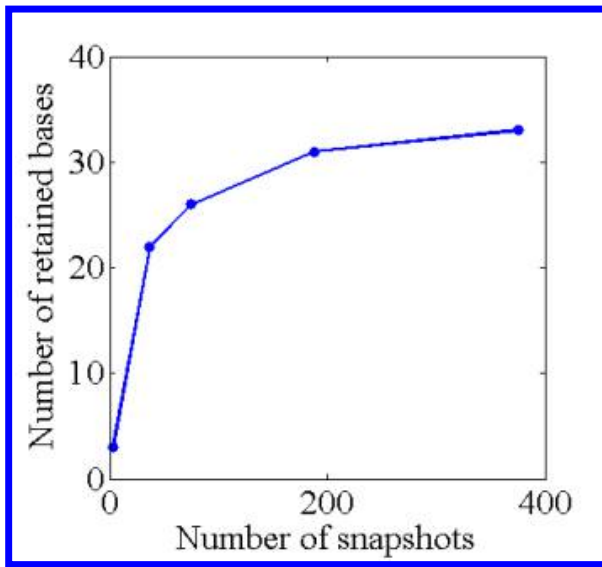

Figure 17: Number of bases to achieve negligible energy loss

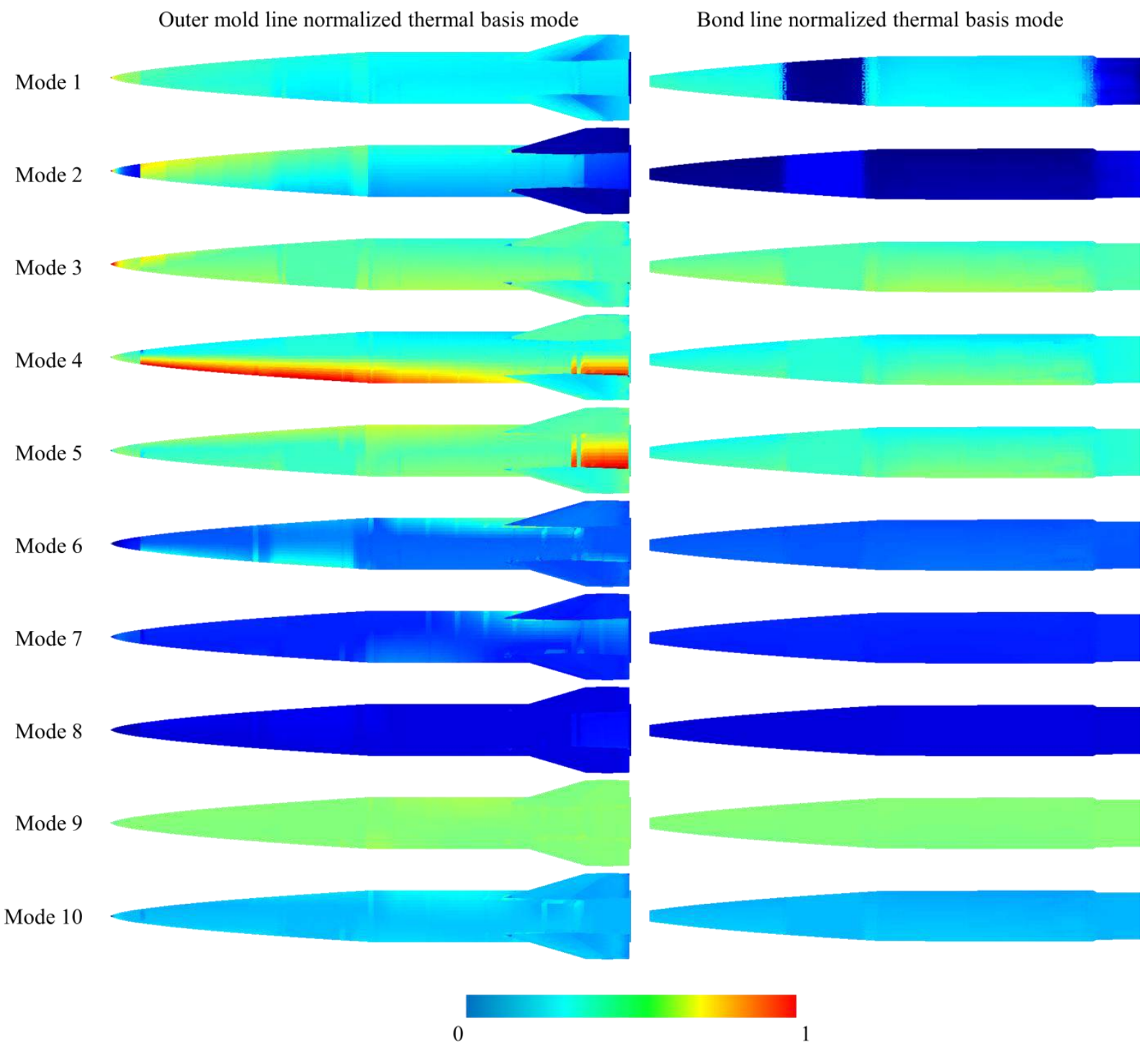

Figure 19: First 10 thermal basis modes normalized by maximum temperature

22

American Institute of Aeronautics and Astronautics

DISTRIBUTION A. Approved for public release, distribution unlimited. (96TW-2015-0158) 


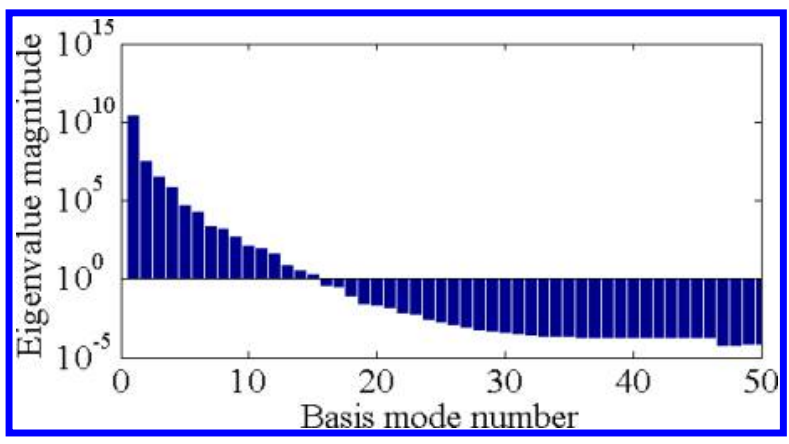

Figure 20: Eigenvalue magnitudes of the first 50 thermal basis modes (0.1-s snapshot sampling)

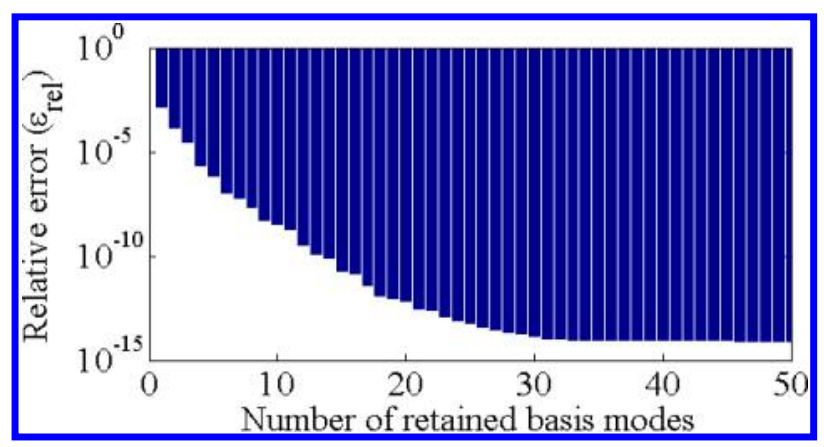

Figure 21: Relative truncation error associated with retaining up to the first 50 thermal basis modes (0.1-s snapshot sampling)

From the OML column in Figure 19, it can be seen that the most dominant thermal basis, mode 1, emphasizes the influence of the relative thermal capacities of the structure near the steel casing, mid-chord of the fins, and TPS near the tail. Mode 2 emphasizes the difference in thermal properties between the ballast and TPS as well as stagnation point heating. Mode 4 emphasizes the difference in temperatures between the windward and leeward sides while modes 8 and 9 almost exclusively focus on fine temperature gradients near the nose stagnation point. From the bond line column, it can be seen that the relatively subtle temperature change of the skin during the terminal phase leads to basis modes that only emphasize the effect of the steel casing in modes 1 and 2 and some slight differences between the windward and leeward sides in modes 3,4 , and 5 .

By projecting the 0.1-s interval snapshot matrix A into the space spanned by the first 33 basis modes $\boldsymbol{\Psi}$, the variation of the amplitude of each mode $\mathbf{c}$ can be seen along the terminal phase of the trajectory. In general, since the basis modes have been truncated, some information has been lost. Therefore, the snapshot matrix will not project perfectly onto the truncated modal space and an error $\underline{\varepsilon}$ can be introduced. However, since it has been shown that projection of the snapshot matrix $\mathbf{A}$ onto 33 basis modes of $\boldsymbol{\Psi}$ produces relative errors on the order of $10^{-15}$, from a numerical standpoint it is possible to project the snapshot matrix directly within computer accuracy, i.e.

$$
\mathbf{c}=\boldsymbol{\Psi}^{-1} \mathbf{A}+\boldsymbol{\varepsilon}
$$

where $\boldsymbol{\varepsilon}$ is approximately 15 orders of magnitude smaller than $\boldsymbol{\Psi}^{-1} \mathbf{A}$ and may be neglected. The coordinates of the first 9 thermal basis modes throughout the terminal phase are shown in Figure 22. Overall, mode 1 dominates throughout much of the terminal phase and begins to yield to mode 2 at $28 \mathrm{~s}$ following the transit to the troposphere and final quenching of the OML. Mode 3 appears to vary in response to a combination of angle of attack and atmospheric conditions. Modes 4, 5, 6, and 7 are more dynamic than the lower modes and are especially active during the pitch-over maneuver at the initiation of the terminal phase. Mode 8 , which contributes almost exclusively to the stagnation point temperature profile, shows the exact time of troposphere transit with a sudden change in slope at $26.4 \mathrm{~s}$. 


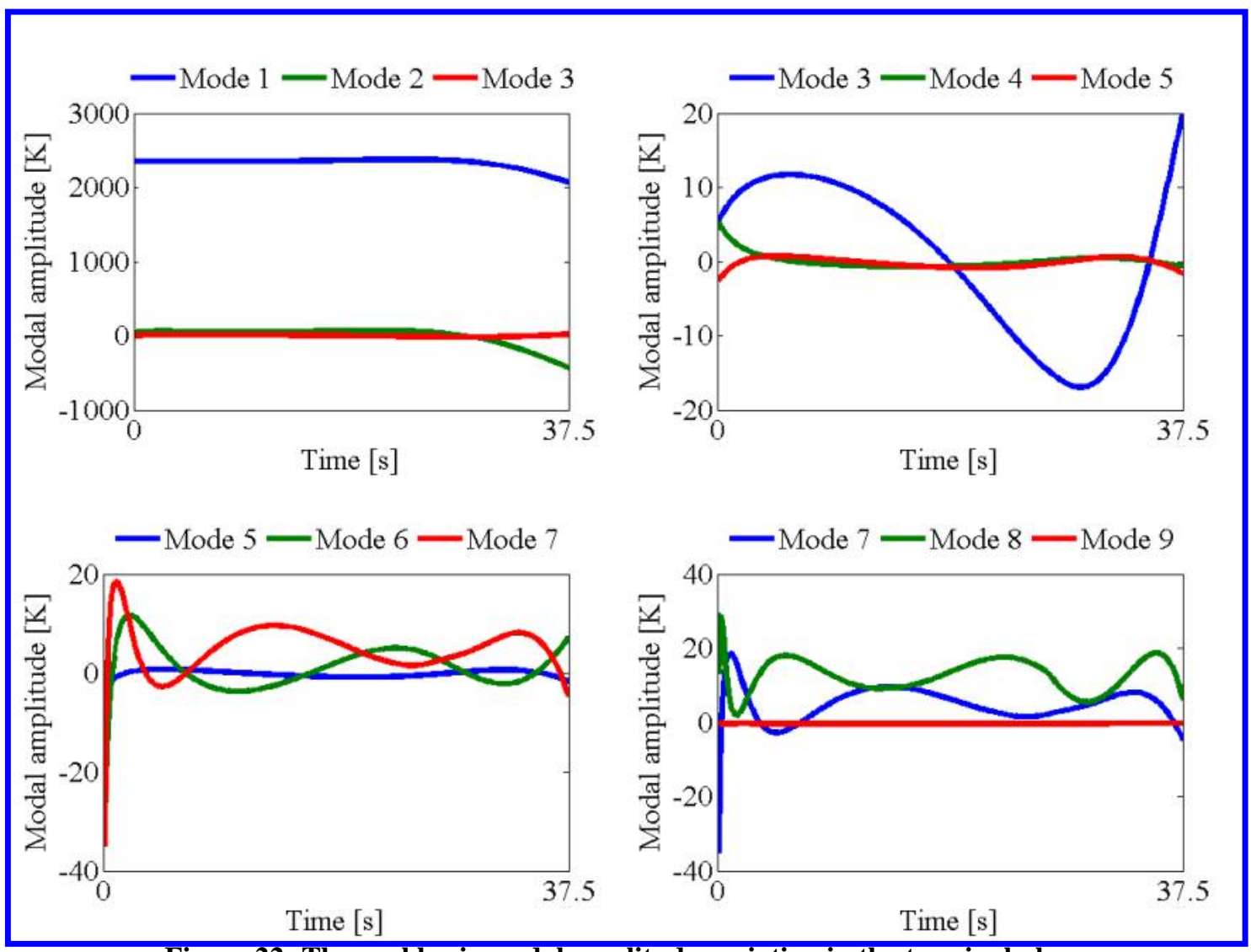

Figure 22: Thermal basis modal amplitude variation in the terminal phase

\section{E. Structural Model Reduction}

To reduce the number of degrees of freedom of the structural model, the structural equations of motion were generalized into a modal form. A common set of mode shapes to use for such generalization are the free vibration mode shapes. However, due to the degradation of the material stiffnesses at elevated temperatures and geometric stiffening of the structure due to thermal gradients and dissimilar interface materials, the normal modes will change in time. Instead, modes determined at a reference thermal state will be used as a set of Ritz modes for model reduction. Thus, a reference thermal state was determined by taking the median temperatures of each FEM node throughout the terminal phase. The median temperatures were considered rather than the mean temperatures since the final quench of the OML skewed the temporal distribution of temperatures toward a lower value that was not representative of the majority of the terminal phase. This reference thermal state is shown in Figure 23.

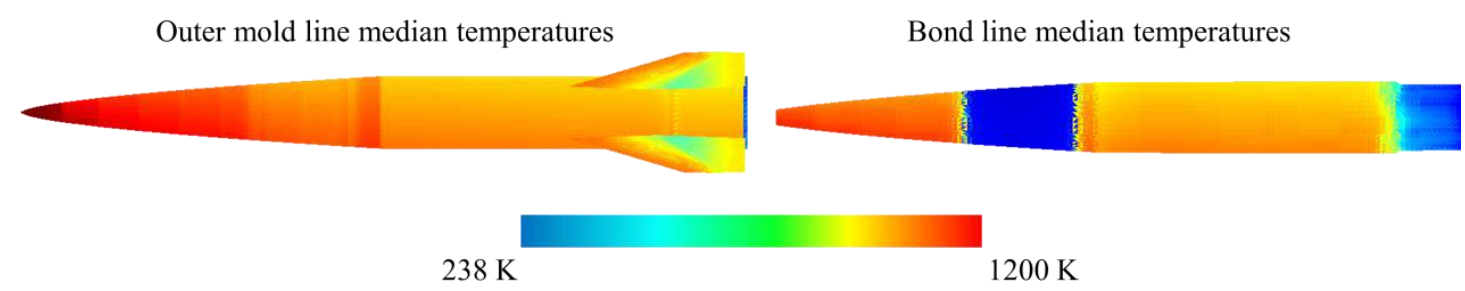

Figure 23: Reference thermal state for structure modal identification

Using the Abaqus ${ }^{30}$ linear perturbation, frequency analysis with the Lanczos ${ }^{32}$ solver, the mass normalized mode shapes and frequencies up to 1 to $200 \mathrm{~Hz}$ were obtained and are shown in Figure 24. The $200 \mathrm{~Hz}$ upper limit was determined by the maximum frequency that could realistically be acted upon by a hypothetical flight controller and fin actuator. Breathing modes of the structure were omitted except in the cases where they were coupled with the first extensional mode. No intermittent contact effects where considered between the fins and the body, which allow

American Institute of Aeronautics and Astronautics

DISTRIBUTION A. Approved for public release, distribution unlimited. (96TW-2015-0158) 
the fins to intersect the body in the cases of the $2^{\text {nd }}$ and $3^{\text {rd }}$ bending as well as the $1^{\text {st }}$ extension modes in case deformation becomes large enough.

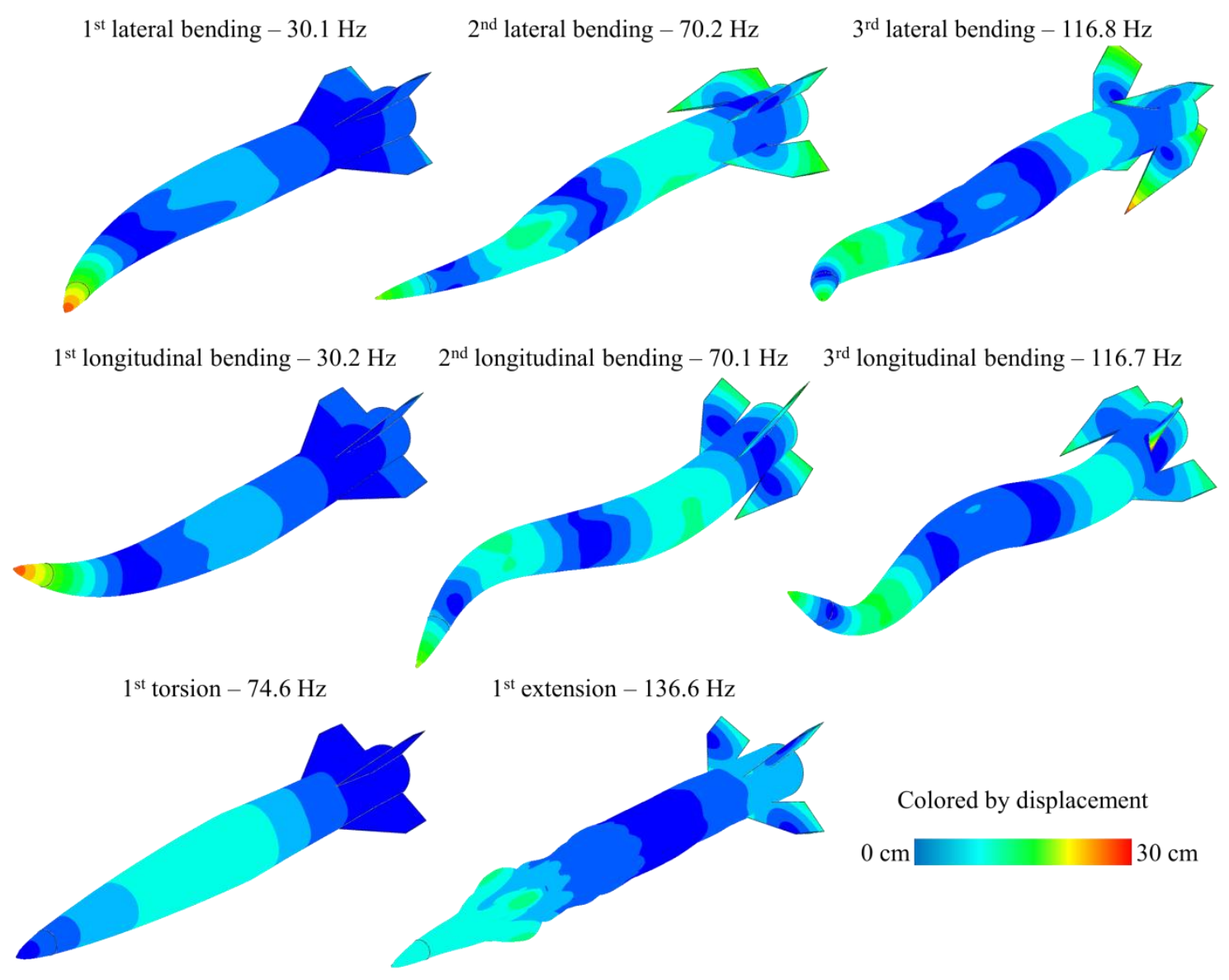

Figure 24: Free vibrational modes at the reference thermal state

\section{F. Thermoelastic Coupling}

While the Ritz modes determined in the previous section are orthogonal at the reference thermal state, there is no guarantee that these modes will remain orthogonal as the stiffness and mass properties vary during flight or along trajectories different than the one selected for this study. The loss of orthogonality is acceptable when integrating the structural equations of motion so long as the variations of the stiffness and mass matrices are modeled. For the terminal phase of the trajectory, it is assumed the engine is off and, therefore, there are no changes to the vehicle mass. For the stiffness part, it is proposed to use Kriging for the approximation of the stiffness properties, as introduced by Falkiewicz and Cesnik. ${ }^{29}$ Development of an appropriate Kriging model would begin with sampling the generalized stiffness matrix entries at various thermal states described by combinations of the POD thermal modes. In Ref. 29, the thermal state was considered as a linear combination of the POD thermal modes and material elastic properties were considered to be dependent on temperature. However, the material thermal conductivities and capacities were taken to be constant. One can see from Figure 5 that at elevated temperatures, material thermal conductivities and capacities are not constant, but vary greatly with temperature; sometimes by $>500 \%$ over certain ranges for the TPS material. This means that the thermal basis functions may not be an accurate representation of the thermal state for large amplitudes or temperature ranges beyond what was considered in the original snapshots. Thus, a nonlinear correction model or thermal mode coordinate mapping should be developed to account for these changes to the thermal properties of the vehicle, prior to development of a stiffness matrix Kriging model.

American Institute of Aeronautics and Astronautics DISTRIBUTION A. Approved for public release, distribution unlimited. (96TW-2015-0158) 


\section{G. Aerothermoelastic Simulation}

The overall goal in the development of the aerodynamic, thermodynamic, structural dynamic, and thermoelastic coupling models described above is the implementation of the representative vehicle model into an in-house aerothermoelastic simulation framework for full six degree-of-freedom flight. This framework was originally developed to demonstrate the partitioned-solution approach to the simulation of supersonic and hypersonic vehicles and is described in detail in Ref. 49. Once the representative vehicle is implemented into this framework, various computational tools already developed may be brought to bear for vehicle trim with thermal and elastic considerations, linearized state-space and control-input matrix identification, static and dynamic stability analysis, and full time-marching flight simulation. With these coupled aerothermoelastic tools, the ultimate goal is to support vehicle design, evaluate flight envelope boundaries with stability, structural, and thermal constraints, and aid in the development and evaluation of control laws for hypersonic vehicles of the type introduced in this study.

\section{Concluding Remarks}

Several reduced order models and basis mode sets were developed for the aerodynamics, thermodynamics, and structural dynamics of a representative hypersonic vehicle. The aerodynamics model was based upon and combination of shock, expansion, and piston (SEP) theories and compared to Euler CFD results with good agreement OML geometry. However some overestimation of pressure at the nose and a lack of flow shadowing were observed.

The aerodynamics model was used to determine lift and drag characteristics of the rigid vehicle which were then used with the GPOPS-II code suite along with 3 degree of freedom equations of motion for longitudinal flight. A set of terminal trajectories ranging in starting conditions from 50 to $100 \mathrm{kft}$ (15.2 to $30.5 \mathrm{~km}$ ) in altitude, Mach 5 to 10 were optimized to deliver maximum final velocity to a ground impact. A representative terminal trajectory starting at $75 \mathrm{kft}(22.9 \mathrm{~km})$ altitude and Mach 6 was selected from the optimized trajectory set for aerothermal analysis. A steady, level cruise trajectory was matched to and preceded the terminal trajectory to establish the initial conditions of the thermal state. During cruise, the vehicle was assumed to vary in trim according to a constant consumption of fuel by an undescribed high-speed propulsion system.

Throughout the cruise and terminal trajectories, aeroheating was modeled using the Eckert reference temperature method with limited real-gas effects. Outer-flow conditions of an assumed fully-turbulent boundary layer were determined using the SEP aerodynamics model. Simulation of the heat transfer within the structure was performed using Abaqus. Following simulation of the heat transfer process, the method of snapshots was used to determine POD thermal bases. Several time intervals between snapshots were compared. It was determined that a 0.1-s snapshot interval was sufficient and resulted in 376 POD thermal modes for the terminal trajectory. Of these modes, 33 were identified and shown to provide an adequate reconstruction of the thermal state with negligible loss of overall system energy when compared to the original snapshots.

To begin modeling the elastic deformation, 8 mass-normalized free vibration modes were determined at a reference thermal state taken to be the temporally median temperature distribution during the terminal trajectory. The median temperature was preferred rather than the mean due to a rapid OML quenching observed in the final seconds of the terminal trajectory. The need to model the variation of the elastic modes due to thermal effects was highlighted. However, development of a thermal model which accounted for the nonlinear effects the material thermal conductivities and capacities would need to be developed prior to developing a model for the variation of the stiffnesses.

Finally, with these models, the representative vehicle may be implemented into an in-house, six rigid degree-offreedom, simulation framework. The ultimate goal is then to enable a fully coupled vehicle simulation, flight envelope identification, and control law development and evaluation.

\section{Acknowledgments}

This work was sponsored by the Air Force Research Laboratory (AFRL), Munitions Directorate, Eglin Air Force Base, Florida. The technical monitor is Dr. Crystal Pasiliao. Opinions, interpretations, conclusions, and recommendations are those of the authors and are not necessarily endorsed by the United States Government.

\section{References}

${ }^{1}$ Pasiliao, C.L., Sytsma, M.J., Neergaard, L.J., Witeof, Z.D., and Trolier, J.W., "Preliminary Aerothermal Structural Simulation", Proceedings of the $14^{\text {th }}$ AIAA Aviation Technology, Integration, and Operation Conference, AIAA 2014-2292.

\section{6}

American Institute of Aeronautics and Astronautics

DISTRIBUTION A. Approved for public release, distribution unlimited. (96TW-2015-0158) 
${ }^{2}$ Witeof, Z.D., and Neergaard, L.J., "Initial Concept 3.0 Finite Element Model Definition," Air Force Research Laboratory, Rept. AFRL-RWWV-TN-2014-0013, Eglin AFB, FL, April 2014. (96ABW-2014-0141).

${ }^{3}$ Trolier, J.W., Hall, D.W., Hudson, D.J., and Torres, M., “Aerothermal Targets Analysis Program (ATAP): Volume 2 - Engineering Document", SAIC-95/1022, 2004.

${ }^{4}$ Zhao, J., and Zhou, R., "Reentry Trajectory Optimization for Hypersonic Vehicle Satisfying Complex Constraints," Chinese Journal of Aeronautics, Vol. 26, No. 6, 2013, pp. 1544 - 1553.

${ }^{5}$ Rao, A.V., and Clarke, K.A., "Performance Optimization of a Maneuvering Re-Entry Vehicle Using a Legendre Pseudospectral Method", Proceedings of the AIAA Atmospheric Flight Mechanics Conference, Monterey, CA, 2002, AIAA 2002-4885.

- ${ }^{6}$ Grant, M.J., and Mendeck, G.F. "Mars Science Laboratory Optimization Using Particle Swarm Methodology", Proceedings of the AIAA Atmospheric Flight Mechanics Conference, Hilton Head, SC, 2007, AIAA $2007-6393$.

${ }^{7}$ Hargraves, C.R., Paris, S.W., and Vlases, W.G., "OTIS Past, Present, and Future”, Boeing Defense and Space Group, Seattle, WA, AIAA 92-4530.

${ }^{8}$ Richardson, D.F., and Rogers, C.E., "Computer Program for Aerospace Trajectory Simulation for Operation on a Personal Computer", Air Force Flight Test Center, 1995.

${ }^{9}$ Zhang, K., and Chen, W., "Reentry Vehicle Constrained Trajectory Optimization", Proceedings of the $17^{\text {th }}$ AIAA International Space Planes and Hypersonic Systems and Technologies Conference, San Francisco, CA, 2011, AIAA 2011-2231.

${ }^{10}$ Mor, M., and Livne, E., "Multidisciplinary Design Optimization of Reentry Vehicles: Trajectory Optimization and Sensitivities", Proceedings of the $47^{\text {th }}$ AIAA/ASME/ASCE/AHS/ASC Structures, Structural Dynamics, and Materials Conference, Newport, RI, 2006, AIAA 2006-1718.

$\checkmark{ }^{11}$ Garg, D., Patterson, M.A., and Darby, C.L., "Direct Trajectory Optimization and Costate Estimation of FiniteHorizon and Infinite-Horizon Optimal Control Problems Using a Radau Pseudospectral Method", Computational Optimization and Applications, Vol. 49, No. 2, 2011, p. 335 - 358.

${ }^{12}$ Rexius, S.L., Rexius, T., Jorris, T.R., and Rao, A.V., "Advances in highly Constrained Multi-Phase Trajectory Generation using the General Pseudospectral Optimization Software (GPOPS)", Proceedings of the AIAA Guidance, Navigation, and Control (GNC) Conference, AIAA 2013-4950.

${ }^{13}$ Richie, G., "The Common Aero Vehicle: Space Delivery System Of The Future", Proceedings of the AIAA Space Technology Conference \& Exposition, Albuquerque, NM, 1999, AIAA 99-4435.

${ }^{14}$ Downey, J.J., and Carr, R.W., "Design of Experiments Using Response Surface Methods to Optimize Simulated Rentry Trajectories", Proceedings of the $28^{\text {th }}$ Aerodynamics Measurement Technology, Ground Testing, and Flight Testing Conference, New Orleans, LA, 2012, AIAA 2012-2726.

${ }^{15}$ Washabaugh, K., Amsallem, D., Zhar, M., and Farhat, C., "Nonlinear Model Reduction for CFD Problems Using Local Reduced Order Bases", Proceedings of the $42^{\text {nd }}$ AIAA Fluid Dynamics Conference and Exhibit, New Orleans, LA, 2012, AIAA 2012-2686.

${ }^{16}$ Lai, K.L., Won, K.S., Koh, E.P.C., and Tsai, H.M., "Flutter simulation and Prediction with CFD-based Reduced-Order Model", Proceedings of the $47^{\text {th }}$ AIAA/ASME/ASCE/AHS/ASC Structures, Structural Dynamics, and Materials Conference, Newport, RI, 2006, AIAA 2006-2026.

${ }^{17}$ Danowsky, B.P., Thompson, P.M., Farhat, C., Lieu, T., Harris, C., and Lechniak, J., "A Complete Aeroservoelastic Model: Incorporation of Oscillation-Reduction-Control into a High-Order CFD/FEM Fighter Aircraft Model", Proceedings of the AIAA Atmospheric Flight Mechanics Conference, Chicago, IL, 2009, AIAA 2009-5708.

${ }^{18}$ Shore, C.P., "Reduction Method for Thermal Analysis of Complex Aerospace Structures", NASA Langley Research Center, TP NASA-TP-2373, 1985.

${ }^{19}$ Craig, R.R., Jr., and Bompton, M.C.C., "Coupling of Substructures for Dynamic Analysis", AIAA Journal, Vol. 6, No. 7, 1968, p. $1313-1319$.

${ }^{20}$ Guyan, R.J., "Reduction of Stiffness and Mass Matrices", AIAA Journal, Vol. 3, No. 2, 1965, p. 380.

${ }^{21}$ Petit, D., Hachette, R., and Veyret, D., "A Modal Identification Method to Reduce a High-Order Model: Application to Heat Conduction Modeling”, International Journal of Modeling and Simulation, Vol. 17, No. 3, 1997, p. $242-250$.

${ }^{22}$ Falkiewicz, N.J., and Cesnik, C.E.S., "Proper Orthogonal Decomposition for Reduced-Order Thermal Solution in Hypersonic Aerothermoelastic Simulations", AIAA Journal, Vol. 49, No. 5, pp. 994-1009.

${ }^{23}$ Liang, Y.C., Lee, H.P., Lim, S.P., Lin, W.Z., Lee, K.H., and Wu, C.G., "Proper Orthogonal Decomposition and its Applications - Part I: Theory", Journal of Sound and Vibration, Vol. 252, No. 3, 2002, p. 527 - 544. 
${ }^{24}$ Bialecki, R.A., Kassab, A.J., and Fic, A., "Proper Orthogonal Decomposition and Modal Analysis for Acceleration of Transient FEM Thermal Analysis", International Journal for Numerical Methods in Engineering, Vol. 62, No. 6, 2005, p. $774-797$.

${ }^{25}$ Bialecki, R.A., Kassab, A.J., and Fic, A., "Reduction of the Dimensionality of Transient FEM Solutions Using Proper Orthogonal Decomposition", Proceedings of the $36^{\text {th }}$ AIAA Thermophysics Conference, Orlando, FL, 2003, AIAA 2003-4207.

${ }^{26}$ Fic, A., Bialecki, R.A., and Kassab, A.J., "Solving Transient Nonlinear Heat Conduction Problems by Proper Orthogonal Decomposition and the Finite-Element Method", Numerical Heat Transfer, Part B: Fundamentals, Vol. 48, No. 2, 2005, p. $103-124$.

$\checkmark{ }^{27}$ Prasad, K.S.R.K., and Murty, A.V. Kristina, "Iterative Type Rayleigh-Ritz Method for Natural Vibration Problems", AIAA Journal, Vol. 8, No. 10, 1970, pp. 1884 - 1886.

${ }^{28}$ Irons, B., "Eigenvalue Economizers in Vibration Problems", Journal of the Royal Aeronautical Society, Vol. 67,1963 , p. $526-528$.

${ }^{29}$ Falkiewicz, N.J, and Cesnik, C.E.S., "Enhanced Modal Solutions for Structural Dynamics in Aerothermoelastic Analysis," 52 $2^{\text {nd }}$ AIAA/ASME/ASCE/AHS/ASC Structures, Structural Dynamics and Materials Conference, Denver, Colorado, 2011, AIAA 2011-1963.

${ }^{30}$ Abaqus FEM/CAE, Ver. 6.12, Dassault Systemes Simulia Corp., Providence, RI, 2012.

${ }^{31}$ Matlab, Ver. 8.1, Rel. 2013a, MathWorks Corp., Natick, MA, 2013.

${ }^{32}$ Cullum, J.K., and Willoughby, R.K., Lanczos Algorithms for Large Symmetric Eigenvalue Computations, Society for Industrial and Applied Mathematics, 1985.

${ }^{33}$ Anderson, Modern Compressible Flow with Historical Perspective, $2^{\text {nd }}$ ed., 2002.

${ }^{34}$ Holt, "Piston Theory - A New Aerodynamics Tool for the Aeroelastician", Journal of the Aeronautical Sciences, Vol. 23, 1956, pp. 1109-1118.

${ }^{35}$ National Aeronautics and Space Administration, U.S. Standard Atmosphere, 1976, Rept. NASA-TM-X-74335.

${ }^{36}$ Saarlas, M., "Reference Temperature Method for Computing Displacement Thickness," AIAA Journal, Vol. 2, No. 11, 1964, pp. 2056-2057.

${ }^{37}$ Arthur, P.D., Schultz, H.D., and Guard, F.L., "Flat Plate Turbulent Heat Transfer at Hypervelocities," Journal of Spacecraft, Vol. 3, No. 10, 1966, pp. 1549-1551.

${ }^{38}$ Crabtree, L.F., Dommett, R.L., and Woodley, J.G., "Estimation of Heat Transfer to Flat Plates, Cones, and Blunt Bodies," Ministry of Technology, Aeronautical Research Council Reports and Memoranda, No. $3637,1970$.

$\checkmark{ }^{39}$ Gunzburger, M. and Peterson, J., "Reduced-Order Modeling of Complex Systems with Multiple System Parameters," Large-Sacel Scientific Computing, Fifth International Conference, edited by Lirkov, I., Margenov, S., and Wasniewski, J., Vol. LNCS 3743, Springer Verlag, 2006, pp. 15-27.

${ }^{40}$ Exelis, "Acusil ${ }^{\circledR}$ II Thermal Protection System," Website: http://www.exelisinc.com/solutions/Acusil\% 20_Thermal_Protection_System/Documents/ACUSIL_II_PR2013.pdf, accessed 7 April 2015.

${ }^{41}$ Ohlhorst, C.W., Vaughn, W.L., Ransone, P.O., and Tsou, H., "Thermal Conductivity Database of Various Structural Carbon-Carbon Composite Materials," Langley Research Center, NASA Technical Memorandum 4787, 1997.

${ }^{42}$ Rehmer, B., Beckmann, J., Finn, M., and Glaubitz, S., "Determination of Elastic Moduli of C/C-Composite at Temperatures up to 1900C," Federal Institute for Materials Research and Testing, Berlin, Germany, 2004.

${ }^{43}$ Fitzer, E., and Manocha, L.M., "Carbon Reinforcements and Carbon/Carbon Composites," Springer-Verlag Berlin Heidelberg, 1998, pp.254-256.

${ }^{44}$ The Engineering Toolbox, "Young Modulus of Elasticity for Metals and Alloys," Website: http://www. engineeringtoolbox.com/young-modulus-d_773.html, accessed 7 April 2015.

${ }^{45}$ The Engineering Toolbox, "Metals - Specific Heats," Website: http://www.engineeringtoolbox.com/specificheat-metals-d_152.html, accessed 7 April 2015.

${ }^{46}$ Allegheny Technologies Incorporated, "ATI Ti-6Al-4V, Grade 5, Technical Data Sheet," ATI Documents Database [online database], accessed 8 April 2015.

${ }^{47}$ Schmidt, F.F., and Ogden, H.R., "The Engineering Properties of Tungsten and Tungsten Alloys," Defense Metals Information Center, Columbus, Ohio, 1963, AD 425547.

${ }^{48}$ Dreyer, E.R., "Fluid-Thermal-Structural Interaction Analyses in Extreme Environments," Proceedings of the $2^{\text {nd }}$ Annual MMOI Meeting, UF REEF, July 2014.

${ }^{49}$ Klock, R.J., and Cesnik, C.E.S., "Aerothermoelastic Simulation of Air-Breathing Hypersonic Vehicles," 55 ${ }^{\text {th }}$ AIAA/ASMe/ASCE/AHS/SC Structures, Structural Dynamics, and Materials Conference, January 2014, AIAA 20140149 . 
This article has been cited by:

1. Ryan J. Klock, Carlos E. S. Cesnik. 2017. Nonlinear Thermal Reduced-Order Modeling for Hypersonic Vehicles. AIAA Journal 55:7, 2358-2368. [Abstract] [Full Text] [PDF] [PDF Plus]

2. Ryan Klock, Carlos E. Cesnik. Nonlinear Thermal and Thermoelastic Reduced Order Models of a Hypersonic Vehicle . [Citation] [PDF] [PDF Plus] 